PALEO

Revue d'archéologie préhistorique

$24 \mid 2013$

Varia

\title{
Les mammouths de Colomb du Pléistocène supérieur de Hot Springs (Dakota du Sud, Etats- Unis)
}

Columbian Mammoths of Late Pleistocene Hot Springs Site (South Dakota, United States)

Sophie Louguet-Lefebvre

URL : http://journals.openedition.org/paleo/2586

DOI : $10.4000 /$ paleo.2586

ISSN : $2101-0420$

Éditeur

SAMRA

Édition imprimée

Date de publication : 15 décembre 2013

Pagination : 149-171

ISSN : 1145-3370

Référence électronique

Sophie Louguet-Lefebvre, "Les mammouths de Colomb du Pléistocène supérieur de Hot Springs (Dakota du Sud, Etats-Unis) », PALEO [En ligne], 24 | 2013, mis en ligne le 17 avril 2014, consulté le 21 décembre 2020. URL : http://journals.openedition.org/paleo/2586 ; DOI : https://doi.org/10.4000/ paleo. 2586

Ce document a été généré automatiquement le 21 décembre 2020.

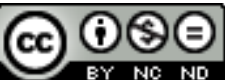

PALEO est mis à disposition selon les termes de la licence Creative Commons Attribution - Pas d'Utilisation Commerciale - Pas de Modification 4.0 International. 


\title{
Les mammouths de Colomb du Pléistocène supérieur de Hot Springs (Dakota du Sud, Etats- Unis)
}

\author{
Columbian Mammoths of Late Pleistocene Hot Springs Site (South Dakota, \\ United States)
}

Sophie Louguet-Lefebvre

Je remercie le docteur Larry D. Agenbroad, professeur émérite en géologie à l'Université de Flagstaff en Arizona et directeur du Mammoth Site, ainsi que toute l'équipe du musée, de m'avoir invitée à étudier cette exceptionnelle collection de fossiles de Proboscidiens et de m'avoir accueillie si chaleureusement, sur le site comme au sein de leur laboratoire de recherche. Un grand merci à Gary Haynes pour sa gentillesse et ses précieuses données. Merci enfin à William Devriendt (cad-dap Douai) pour cette nouvelle illustration, ici la reconstitution de Hot Springs (fig. 2).

\section{1 - Introduction}

Découvert fortuitement en 1974 lors de travaux d'aménagement d'élargissement d'un lotissement, le site paléontologique de Hot Springs dans le Dakota du Sud fut entièrement préservé. Si d'autres sites ont révélé des vestiges de mammouths dans la région, tel le site paléolithique supérieur de Lange Fergusson également mondialement connu (deux mammouths dépecés et associés à des pointes de Clovis au sein d'un ancien marécage, Hannus 1989), le site de Hot Springs est sans conteste le plus impressionnant à en juger par la concentration de squelettes de mammouths in situ révélée (fig. 1). En effet, le site témoigne d'une importante accumulation de vestiges ayant appartenu à une faune typique du Pléistocène supérieur, très largement dominée 
par le mammouth de Colomb qui sillonnait les grandes plaines d'Amérique du Nord il y $\mathrm{a} \pm 26000$ ans BP (moyenne des datations ${ }^{14} \mathrm{C}$, Laury 1990).

2 Le plan d'eau alimenté par une source chaude et la végétation bordière verdoyante, typique d'un marécage, devaient être particulièrement attrayants en période glaciaire pour les animaux et d'autant plus pour les mammouths dont les besoins quotidiens devaient avoisiner un minimum de 200 litres d'eau. La source devait constituer un piège redoutable pour les animaux qui s'aventuraient dans ce marais aux berges abruptes et glissantes se terminant en entonnoir dans sa partie submergée. Ainsi, nombre d'animaux se sont laissés prendre au piège qui s'est avéré mortel pour les mégaherbivores tels les mammouths.

3 Connaître l'origine d'un assemblage osseux d'éléphantidés est loin d'être chose aisée. Ainsi, le recours à un solide référentiel en terme de mortalité naturelle, excluant le facteur anthropique, est capital pour l'analyse des vestiges issus de sites paléontologiques. Cela passe par la connaissance des structures sociales de populations d'éléphants actuels ainsi que par les profils de mortalité dressés à partir d'espèces fossiles issues de sites purement paléontologiques. L'objectif de ce travail est d'appréhender les circonstances possibles de la mort des mammouths sur le site compte tenu de l'analyse du profil de mortalité nouvellement dressé.

Figure 1 - Localisation des principaux sites à Mammuthus columbi.

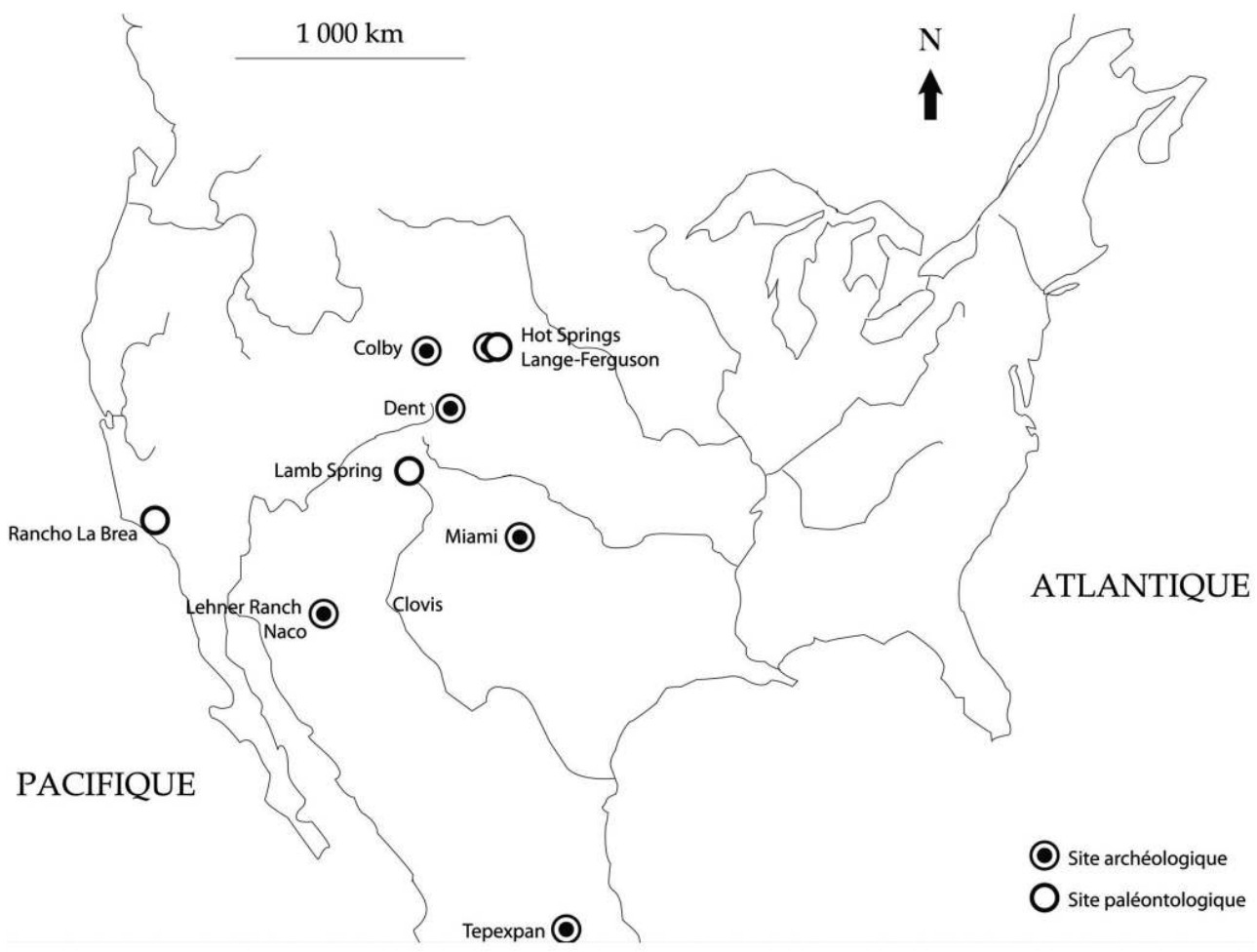

\section{2 - Présentation du site}

\section{1 - Localisation et contexte géologique}

4 Le site est localisé au sud des collines des Black Hills (GPS 43.4291, -103.494774). Il est né de l'effondrement il y a \pm 26000 ans BP (Laury 1990) du plafond d'une caverne 
souterraine (fig. 2). La dépression elliptique de $40 \mathrm{~m}$ de long ainsi formée présentait une paroi dont l'inclinaison variait de $60^{\circ}$ à l'horizontale et une profondeur variant de $14 \mathrm{~m}$ pour la portion sud-ouest à $21 \mathrm{~m}$ dans sa partie nord-est. Une source artésienne chaude y alimenta avec le temps un lac de surface dont la température avoisinait les 35 à $40^{\circ} \mathrm{C}$, comme l'ont attesté les analyses malacologiques, polliniques et ichtyologiques (Agenbroad 1990).

\section{2 - Stratigraphie}

Le remplissage stratigraphique de la cuvette, née de l'effondrement karstique, consiste en une superposition de couches laminées de sédiments à granulométrie fine, allant de couches d'argile à des couches de sable grossier résultant de l'érosion et de la désagrégation de la paroi rocheuse, ainsi que des graviers constitutifs de l'ancienne terrasse de la paléorivière avoisinante. Les vestiges osseux ont été exhumés des trois grandes phases de sédimentation de la séquence, et si la fouille est encore en cours, les principales zones ont d'ores et déjà été sondées en profondeur. Le piège aurait opéré durant environ 300 à 700 ans avant son comblement total par les sédiments de parois (Laury 1990).

6 Le flottement des carcasses en décomposition et les mouvements de gravité subaquatiques sont des facteurs à ne pas négliger dans les processus de dépôts des vestiges osseux. En effet, ceux-ci ont activement contribué à la désarticulation et à l'emplacement spécifiques de certains spécimens. Quelle que soit la phase de sédimentation considérée, la densité des vestiges la plus notable se situe le long des anciennes berges. Les ossements, tous enregistrés en trois dimensions, sont concentrés à moins de $6 \mathrm{~m}$ de la paroi (Laury 1990, fig. 2). Il est possible que le courant artésien ait contribué à dénuer de vestiges la partie centrale du remplissage. La portion nord-est du dépôt présente une forte concentration de vestiges osseux isolés provenant d'une matrice sableuse, conséquence du courant artésien, tandis que la partie sud-ouest présente des éléments en connexion anatomique figés dans un contexte laminé de couches d'argiles et de limons, suggérant une énergie subaquatique nettement plus modérée. Ainsi, la plupart des carcasses étaient partiellement désarticulées avant d'être entraînées vers le bas de la berge immergée puis enfouies sous les sédiments. Le spécimen le plus remarquable mis au jour est le squelette post-crânien complet d'une femelle adulte préservé in situ en connexion anatomique ( $\left.n^{\circ} 13523 \mathrm{~W}\right)$.

\section{3 - Conservation des vestiges}

7 L'érosion progressive des berges a permis l'excellente conservation de l'ensemble des os. Même les plus petits et les plus fragiles, tels que les os hyoïdes ou les sésamoïdes, ont été préservés par le recouvrement rapide par les sédiments des éléments anatomiques tout juste décomposés. Par ailleurs, l'origine naturelle de la fracturation sur os frais, sans impact ni contrecoup, mise en évidence sur onze ossements d'éléphantidés, semble faire peu de doutes. Ceci est corroboré par l'absence de témoignage anthropique sur le site, comme l'avait déjà conclu L.D. Agenbroad (1989). Il est envisageable que des os encore frais aient cassé sous le poids des mammouths mis en difficulté au niveau des berges. 


\section{4 - Liste faunique}

8 Si l'écrasante surreprésentation de mammouths de Colomb (Mammuthus columbi) tend à faire oublier la diversité faunique attestée à Hot Springs, nombre d'espèces animales appartenant aux mammifères, oiseaux, poissons, amphibiens et invertébrés a d'ores et déjà été inventorié (Agenbroad, Mead 1994, réactualisé sur http:// www.mammothsite.com).

9 Parmi les autres grands mammifères présents sur le site, ont été mis au jour le mammouth laineux (Mammuthus primigenius), l'ancien chameau (Camelops hesternus), le lama (Hemiauchenia sp.), l'antilope d'Amérique (Antilocapra americana), et finalement cinq carnivores : l'ours géant à face-courte (Arctodus simus), le loup (Canis lupus), le coyote (Canis latrans), le blaireau (Taxidea taxus) ainsi qu'un autre mustélidé de moindre taille de type vison ou furet (Mustela sp.). Deux espèces de lagomorphes ont aussi été exhumées, à savoir le lièvre de Townsend (Lepus cf. townsendii) et un lapin (Sylvilagus sp.). Les insectivores sont eux représentés par la taupe (Scalopus aquaticus), tandis que nombre de rongeurs viennent enrichir de façon conséquente la liste faunique. Ces derniers comptent tout d'abord le chien de prairie (Cynomys (Leucocrossuromys) sp.), le spermophile rayé (Spermophilus cf. tridecemlineatus) ainsi qu'un écureuil un peu plus grand (Spermophilus cf. elegans ou richardsonii), suivis du gaufre gris (Thomomys talpoides), d'un hamster (Neotoma cf. cinerea), d'une souris (Peromyscus cf. maniculatus), du campagnol des bruyères (Phenacomys cf. intermedius) et d'un campagnol plus petit (Microtus sp.).

10 S'il parait exceptionnel d'avoir mis au jour Mammuthus primigenius (représenté par trois individus) et Mammuthus columbi sur un même site, cela n'implique pas forcément que les deux espèces aient cohabité. En effet, les vestiges osseux de mammouths laineux proviennent de la partie supérieure des sédiments. Cela pourrait indiquer qu'ils aient succédé au mammouth de Colomb sur le site (Agenbroad, Mead 1994). 
Figure 2 - Reconstitution de la source de Hot Springs, \pm 26000 ans BP (๑ W. Devriendt).

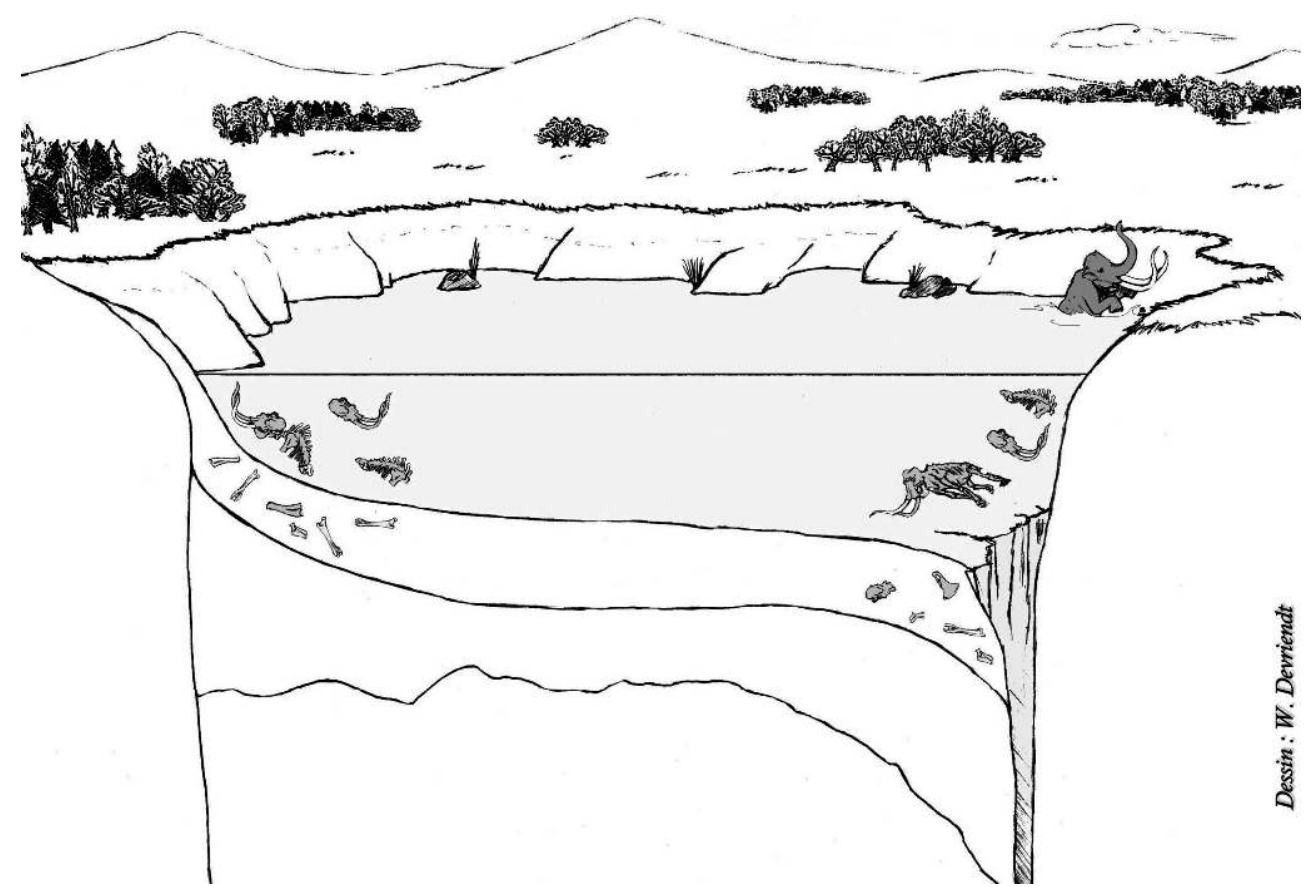

\section{3 - Analyse paléo-environnementale}

11 L'étang, alimenté par la source chaude, était situé non loin de la limite sud de l'inlandsis nord-américain. Sa végétation bordière verdoyante, typique d'un marécage, devait être des plus attrayante pour la faune. Les alentours étaient eux occupés par de grandes étendues de végétation basse, de type steppique, caractéristiques du climat relativement froid environnant, au détriment de la forêt ne persistant qu'à titre résiduel au fond des vallées.

Des méthodes écologiques, basées sur la présence des mammifères, ont été appliquées ici afin d'approcher au mieux la réalité de l'environnement du site à l'époque. Ces méthodes, histogrammes écologiques et cénogramme, ont l'avantage de ne pas tenir compte de la quantification des espèces mais uniquement de leur présence. Le recours à ces outils est donc particulièrement approprié à Hot Springs, qui est encore en cours de fouille et dont la dynamique sédimentaire complexe n'exclut pas une répartition des vestiges par format taxinomique.

\section{1 - Histogrammes écologiques}

La méthode consistant à dresser et à interpréter différents histogrammes écologiques tient compte de la systématique de chaque espèce, mais également de la masse, du régime alimentaire et de l'adaptation locomotrice de chacune d'entre elles. Définie par T.H. Fleming sur les communautés mammaliennes actuelles d'Amérique centrale et d'Amérique du Nord (Fleming 1973), la méthode a ensuite été transposée aux espèces fossiles par P. Andrews et al. (1979), puis modifiée par Cl. Guérin et M. Faure (1987).

La prédominance des rongeurs à Hot Springs, suivis dans une moindre mesure des carnivores et des artiodactyles, caractérise un milieu à dominante forestière. Toutefois, 
l'hégémonie des herbivores aux dents hypsodontes traduit quant à elle l'ouverture du milieu (tabl. 1 , fig. 3 et $4 a$ et b). Cette mixité du couvert végétal est finalement renforcée par l'importance des espèces de moins d'un kilo, suivies dans une moindre mesure par les moins de $10 \mathrm{~kg}$, couplée à la présence remarquée des espèces de plus de $200 \mathrm{~kg}$ (fig. 4c).

Tableau 1 - Légende des histogrammes écologiques de Hot Springs.

\begin{tabular}{|c|c|c|c|c|}
\hline Espèces & Systématique & Masses & Alimentation & Locomotion \\
\hline Mammuthus colombi & Pro & $\mathrm{H}$ & $\mathrm{HH}$ & GT u \\
\hline Mammuthus primigenius & Pro & $\mathrm{H}$ & $\mathrm{HH}$ & GT u \\
\hline Arctodus simus & $\mathrm{C}$ & G & $\mathrm{O}$ & GT u \\
\hline Camelops hesternus & $\mathrm{Ar}$ & $\mathrm{G}$ & $\mathrm{HB}$ & GT u \\
\hline Hemiauchenia sp. & Ar & E & $\mathrm{HB}$ & GT u \\
\hline Canis lupus & $\mathrm{C}$ & $\mathrm{D}$ & $\mathrm{C}$ & GT u \\
\hline Antilocapra Americana & Ar & $\mathrm{D}$ & $\mathrm{HH}$ & GT c \\
\hline Canis latrans & $\mathrm{C}$ & $\mathrm{D}$ & $\mathrm{C}$ & GT u \\
\hline Taxidea taxus & $\mathrm{C}$ & $\mathrm{C}$ & $\mathrm{O}$ & GT u \\
\hline Lepus cf. townsendii & A & $\mathrm{C}$ & $\mathrm{HH}$ & PT \\
\hline Cynomys (Leucocrossuromys) sp. & $\mathrm{R}$ & $\mathrm{C}$ & $\mathrm{HB}$ & PT \\
\hline Mustela cf. vison or nigripes & $\mathrm{C}$ & $\mathrm{C}$ & $\mathrm{O}$ & GT u \\
\hline Sylvilagus sp. & A & $\mathrm{AB}$ & $\mathrm{HH}$ & PT \\
\hline Spermophilus ef. elegans or richardsonii & $\mathrm{R}$ & $\mathrm{AB}$ & $\mathrm{F}$ & $\mathrm{Gr}-\mathrm{Ar}$ \\
\hline Neotoma cf. cinerea & $\mathrm{R}$ & $\mathrm{AB}$ & $\mathrm{F}$ & Fo \\
\hline Spermophilus cf. tridecemlineatus & $\mathrm{R}$ & $\mathrm{AB}$ & $\mathrm{F}$ & $\mathrm{Gr}-\mathrm{Ar}$ \\
\hline Thomomys talpoides & $\mathrm{R}$ & $\mathrm{AB}$ & $\mathrm{HH}$ & Fo \\
\hline Scalopus aquaticus & $\mathrm{I}$ & $\mathrm{AB}$ & $\mathrm{I}$ & Fo \\
\hline Microtus sp. & $\mathrm{R}$ & $\mathrm{AB}$ & $\mathrm{HH}$ & Fo \\
\hline Phenacomys cf. intermedius & $\mathrm{R}$ & $\mathrm{AB}$ & $\mathrm{HH}$ & Fo \\
\hline Peromyscus cf. maniculatus & $\mathrm{R}$ & $\mathrm{AB}$ & $\mathrm{F}$ & Fo \\
\hline
\end{tabular}

$\mathrm{R}=$ rongeurs $; \mathrm{I}=$ insectivores $; \mathrm{Pri}=$ primates $; \mathrm{Ar}=$ artiodactyles $; \mathrm{C}=$ carnivores $; \mathrm{Per}=$ périssodactyles Pro $=$ proboscidiens $; \mathrm{A}=$ autres ordres, ici lagomorphes

$\mathrm{AB}<1 \mathrm{~kg} ; \mathrm{C}=1-10 \mathrm{~kg} ; \mathrm{D}=10-45 \mathrm{~kg} ; \mathrm{E}=45-100 \mathrm{~kg} ; \mathrm{F}=100-200 \mathrm{~kg} ; \mathrm{G}=200-1000 \mathrm{~kg} ; \mathrm{H}=>\mathrm{It}$

$\mathrm{I}=$ insectivores $; \mathrm{F}=$ frugivores et granivores $; \mathrm{HB}=$ herbivores brachyodontes $; \mathrm{HH}=$ herbivores hypsodontes $; \mathrm{C}=$ carnassiers $; \mathrm{O}=$ omnivores

GTf, GTu, GTc = grands mammifères terrestres forestiers, ubiquistes, coureurs ; PT = petits mammifères terrestres ; $\mathrm{Gr}-\mathrm{Ar}=$ grimpeurs et arboricoles $; \mathrm{Aq}=$ aquatiques $; \mathrm{Ae}=$ aériens, $\mathrm{Fo}=$ fouisseurs 
Figure 3 - Profils d'histogrammes écologiques théoriques, d'après S. Péan 2001 (cf. légende tableau 1).

a/ Systématique

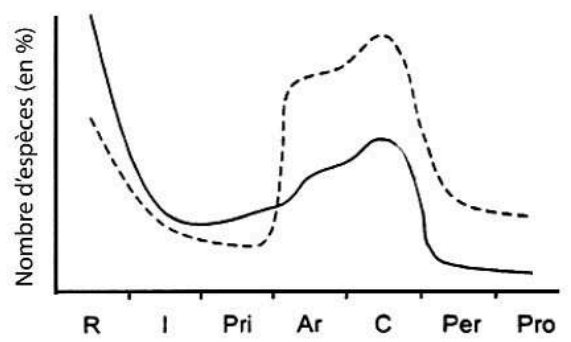

c/ Masse

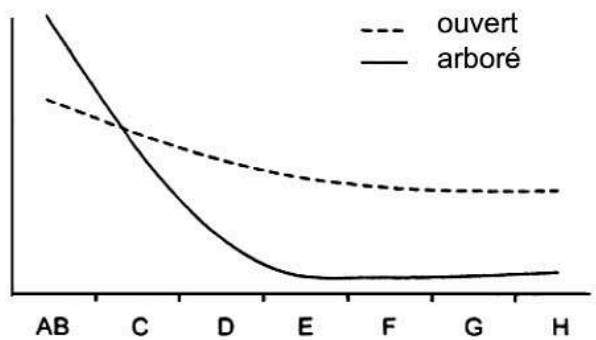

b/ Régime alimentaire

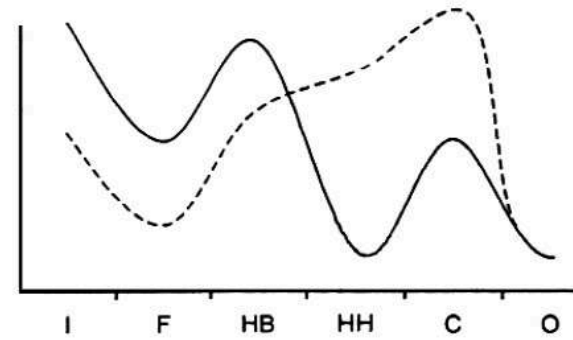

d/ Locomotion

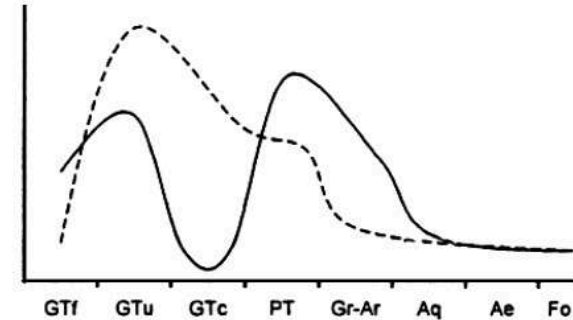

Figure 4 - Histogrammes écologiques de Hot Springs (cf. légende tableau 1).

a/ Systématique

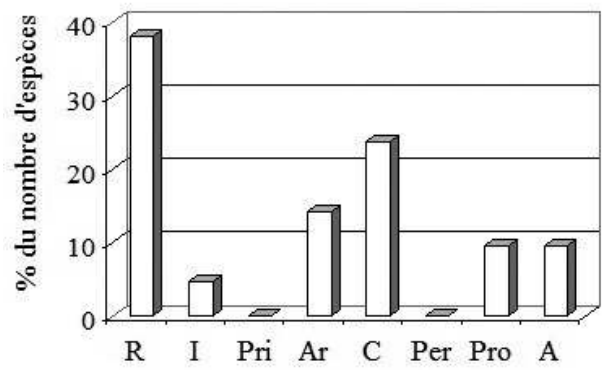

c/ Masse

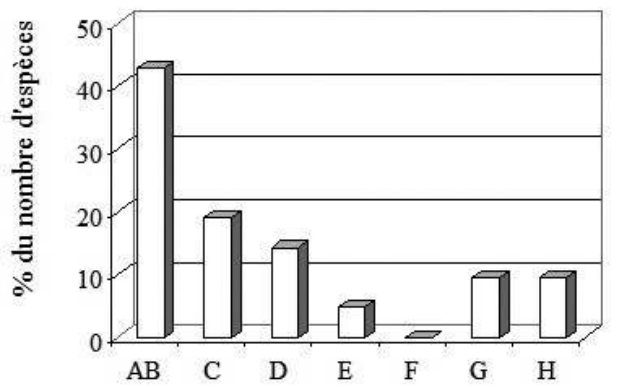

b/ Régime alimentaire

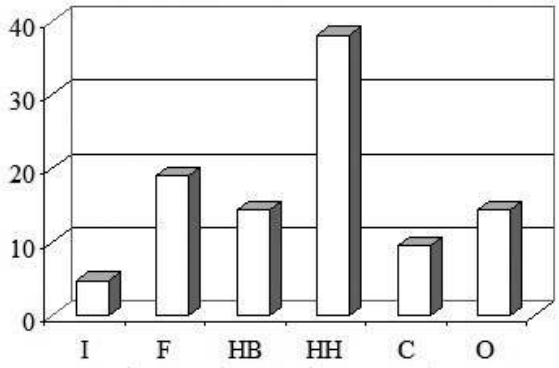

d/ Locomotion

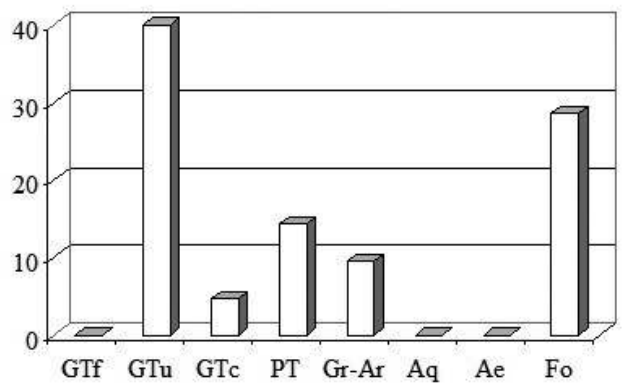




\section{2 - Cénogramme}

L'étude effectuée par S. Legendre sur les faunes actuelles a mis en évidence une forte corrélation entre la distribution pondérale des mammifères et leur environnement. La méthode des cénogrammes de J.A. Valverde (1964) modifiée par S. Legendre a consisté à reporter graphiquement en abscisse les différents taxons, hors carnivores, classés par ordre décroissant de masse, et en ordonnée le logarithme de leur masse exprimée en grammes. L'estimation de la masse moyenne de la plupart des espèces fossiles a été effectuée par $\mathrm{S}$. Legendre en fonction de la surface de leur première molaire inférieure, selon une formule spécifique et des constantes propres aux groupements taxinomiques (Legendre 1989). Concernant la masse approximative de certaines espèces d'autres références ont été considérées (tabl. 2).

Tableau 2 - Légende du cénogramme de Hot Springs.

\begin{tabular}{|c|c|c|c|c|}
\hline Espèces & Rang & $\begin{array}{l}\text { Estimation } \\
\text { masse }(\mathrm{kg})\end{array}$ & $\begin{array}{r}\log \mathrm{Y} \\
(\text { masse en } \mathrm{g})\end{array}$ & Référence \\
\hline Mammuthus colombi & 1 & 8000 & 15,8949 & Agenbroad, Mead 1994 \\
\hline Mammuthus primigenius & 2 & 6000 & 15,6073 & Agenbroad, Mead 1994 \\
\hline Arctodus simus & - & 600 & - & Figueirido et al. 2010 \\
\hline Camelops hesternus & 3 & 570 & 13,25339 & actuelle Legendre 1989 \\
\hline Hemiauchenia sp. & 4 & 92 & 11,43 & actuelle Legendre 1989 \\
\hline Canis lupus & - & 45 & - & Legendre 1989 \\
\hline Antilocapra americana & 5 & 39,6 & 10,587 & Legendre 1989 \\
\hline Canis latrans & - & 13,4 & - & Legendre 1989 \\
\hline Taxidea taxus & 6 & 6 & 8,68101 & Legendre 1989 \\
\hline Lepus cf. townsendii & 7 & 1,5 & 7,313 & Legendre 1989 \\
\hline Cynomys (Leucocrossuromys) sp. & 8 & 1,25 & 7,131 & actuelle \\
\hline Mustela cf. vison/nigripes & - & 1 & - & Aulagnier et al. 2010 \\
\hline Sylvilagus sp. & 9 & 0,95 & 6,86 & actuelle Legendre 1989 \\
\hline Spermophilus cf. elegans/richardsonii & 10 & 0,32 & 5,75574 & Legendre 1989 \\
\hline Neotoma cf. cinerea & 11 & 0,315 & 5,75257 & actuelle \\
\hline Spermophilus cf. tridecemlineatus & 12 & 0,165 & 5,106 & Legendre 1989 \\
\hline Thomomys talpoides & 13 & 0,105 & 4,654 & actuelle \\
\hline Scalopus aquaticus & 14 & 0,058 & 4,06 & Legendre 1989 \\
\hline Microtus sp. & 15 & 0,04 & 3,663 & actuelle Legendre 1989 \\
\hline Phenacomys cf. intermedius & 16 & 0,035 & 3,5553 & actuelle \\
\hline Peromyscus cf. maniculatus & 17 & 0,019 & 2,97 & Legendre 1989 \\
\hline
\end{tabular}

Tandis que les espèces comprises entre $500 \mathrm{~g}$ et $5 \mathrm{~kg}$ sont abondantes en milieu fermé forestier, elles sont absentes ou exceptionnelles en milieu ouvert steppique, se traduisant par une rupture de pente sur le cénogramme (fig. 5). Trois taxons parmi les dix-sept, hors carnivores, recensés à Hot Springs rentrent dans cette fourchette pondérale, à savoir le lièvre, le lapin et le chien de prairie (annexe 2, fig. 6). Leur présence suggère l'existence d'un milieu mixte ou la forêt n'est pas en reste. Par ailleurs, plus le contexte est humide plus la diversité écologique est favorisée, d'où une pente plus régulière que dans le cas d'un environnement semi-désertique (Legendre 1989). La pente est dans notre cas irrégulière et la liste d'espèces mammaliennes plutôt restreinte à ce jour. Toutefois, une aridité excessive aurait vraisemblablement limité la présence des très grandes espèces, ce qui n'est guère le cas ici. 
Figure 5 - Interprétations des cénogrammes, d'après Montuire, Marcolini 2002.

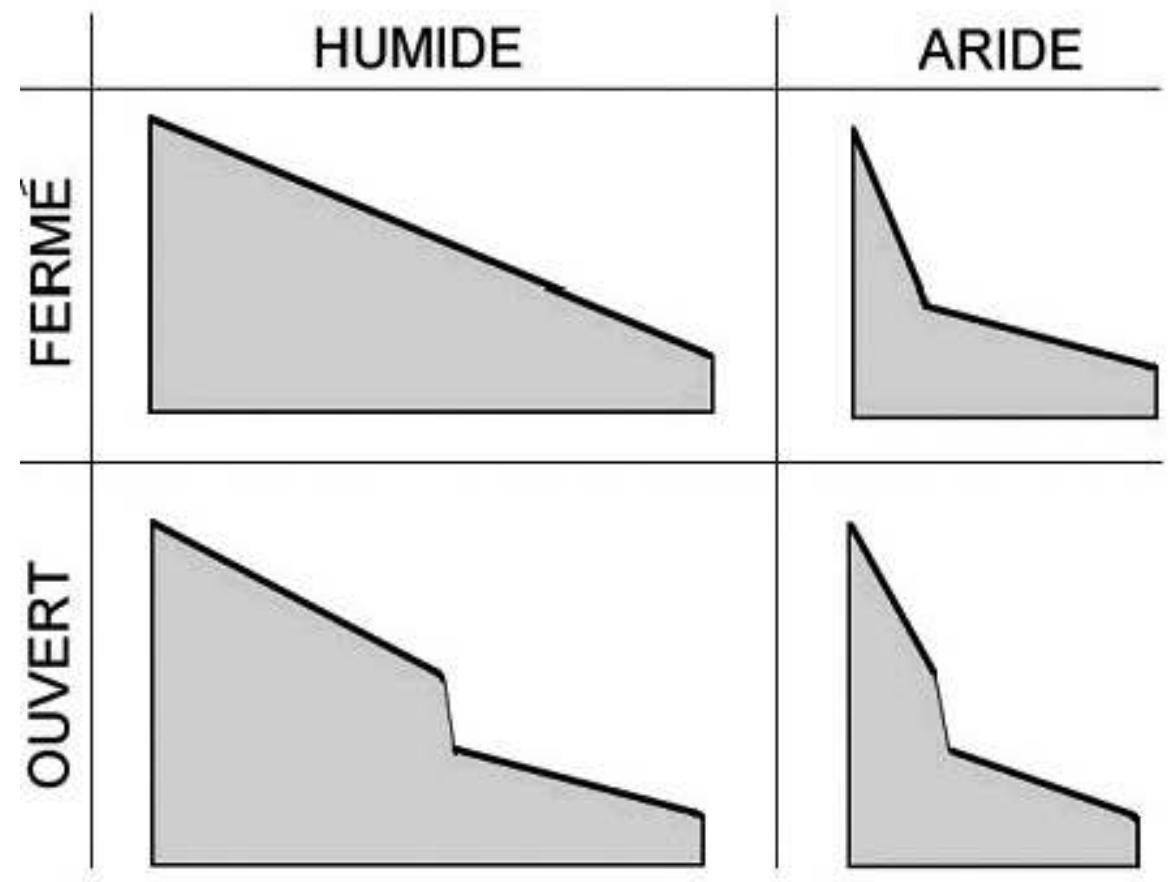

Figure 6 - Cénogramme de Hot Springs.

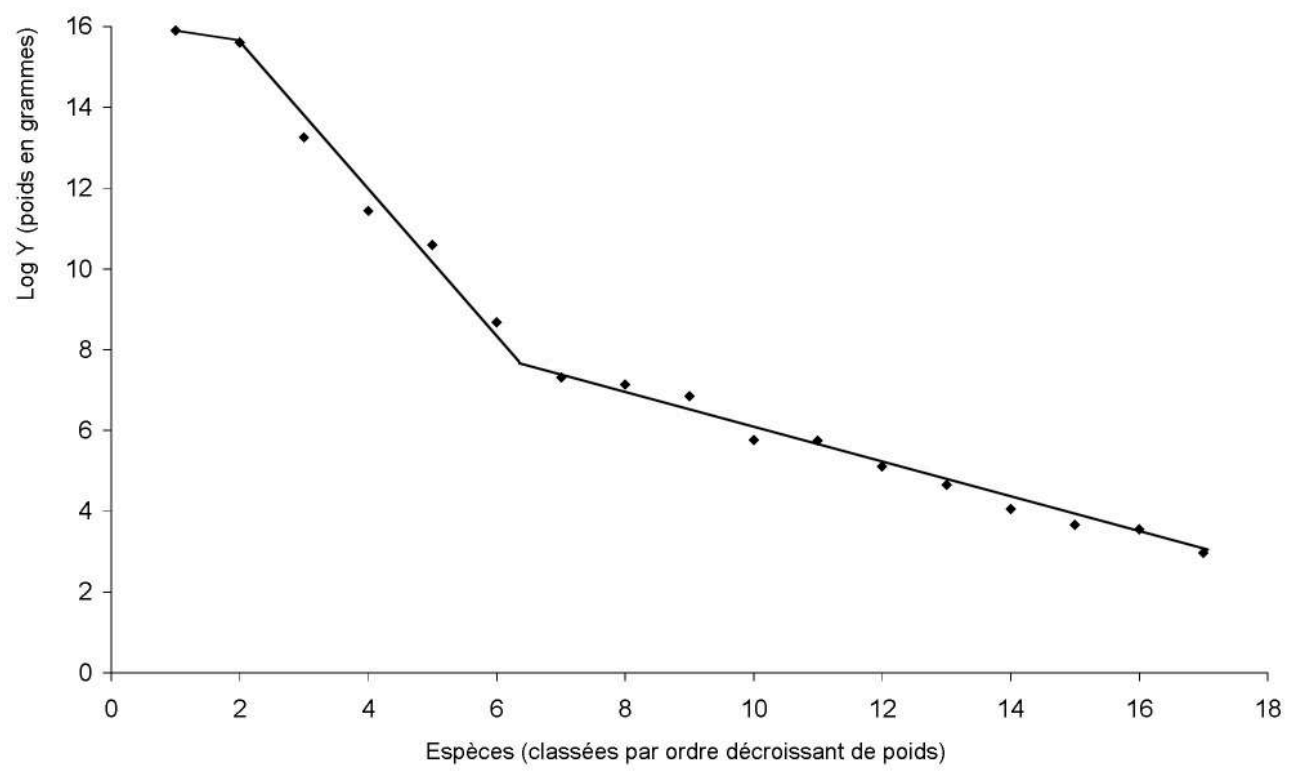

\section{3 - Interprétation paléo-environnementale}

17 Histogrammes écologiques et cénogramme de Hot Springs tendent à caractériser un milieu mixte immédiat mêlant la steppe et la forêt sous un climat globalement subaride. Nos résultats sont en adéquation avec l'ensemble des données paléoenvironnementales qui caractérise un îlot de verdure au cœur de la steppe (Mead et al. 1990). L'analyse palynologique indique ainsi que $46 \%$ des pollens proviennent de 
graminées (Poacea), même si sont aussi présentes les plantes de milieux humides de type laîches (Carex), les plantes aquatiques (Potamogeton, Polygonum), les plantes aromatiques (Artemisia) ou les plantes à fleurs (Ambrosia, Chenopodiaceae). Quant aux espèces arboricoles, moins abondantes, l'analyse palynologique indique la présence de l'épicéa suivi de feuillus (bouleau, genévrier, noyer, orme, chêne).

\section{4 - Révision du profil de mortalité des mammouths : matériel et méthodes}

\section{1 - Présentation des vestiges crâniens de mammouths de Colomb}

18 Parmi les squelettes de Mammuthus columbi présentant des connexions anatomiques, le plus complet est sans conteste celui qui a pour numéro HS00140, sa tête étant demeurée en connexion étroite avec le reste du corps (fig. $7 \mathrm{~d}$ ). Le spécimen $13 \mathrm{~S} 23 \mathrm{~W}$, attribué par A. Lister à une femelle, présente quant à lui un squelette post-crânien in situ quasi-complet. Le crâne 87-016 pourrait y être associé compte tenu de sa proximité avec ce dernier et l'écartement de ses condyles occipitaux (Lister in Agenbroad, Mead 1994).

Figure 7 - Vestiges crâniens de Mammuthus columbi, Hot Springs (a/ crâne $n^{\circ} \mathrm{HS00147}$; b/ mâchoire supérieure $n^{\circ} 79.265 ; c /$ mandibule $n^{\circ}$ HSO0283; d/ mandibule $n^{\circ}$ HSO0140).

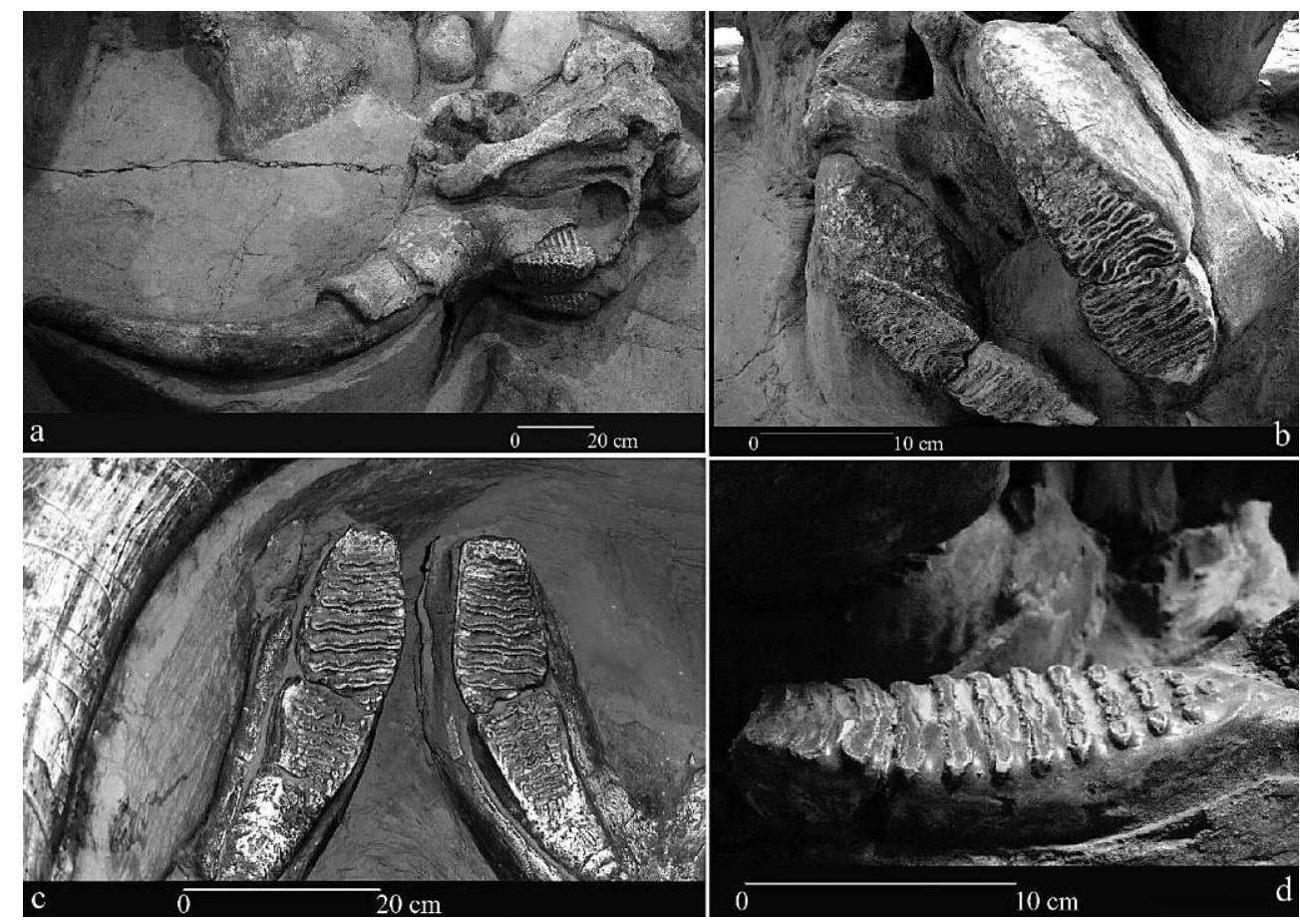

19 Parmi les vingt-trois crânes étudiés par L.D. Agenbroad en 1994, neuf n'avaient pas permis l'attribution d'un âge, contre une mandibule parmi les douze prises en considération à l'époque (Agenbroad, Mead 1994). Parmi les quarante-huit mâchoires totalisées fin 2005, sept ne nous permirent pas d'accéder aux molaires. Seuls quatre crânes sur les vingt-quatre examinés et trois mandibules sur vingt-quatre une fois encore ne nous donnèrent pas accès à la dentition (tabl. 3 et 4 - en annexe). Autant de mâchoires supérieures que de mandibules ont donc été prises en compte sans que l'on 
sache s'il est possible ou non de les associer. Cent onze dents jugales analysées appartiennent ainsi à des séries dentaires (deux dents consécutives, exceptionnellement trois) contre quarante-six molaires isolées, soit un effectif total de cent cinquante-sept jugales. Depuis fin 2005, plusieurs éléments post-crâniens ont été exhumés. Toutefois, hormis les défenses, seul un crâne supplémentaire a été mis au jour au cours de la fouille de 2009 (http://www.mammothsite.com).

Deux mandibules difficilement accessibles ainsi que vingt-cinq vestiges dentaires isolés sont d'âge indéterminé. Il s'agit dans treize cas de fragments de molaires constitués de quelques lames et dans six cas de lames uniques. Dans le cas de quatre molaires entières mais en partie masquées par le sédiment, c'est le rang incertain de la dent au sein de la série jugale qui ne suffit qu'à lui attribuer une possibilité d'appartenance (par exemple M2 ou M3). Une dent en fin de vie est finalement si abrasée que son appartenance est également sujette à caution. Parmi les molaires entières, on notera que l'une avoisine les six ou quinze ans tandis que les autres proviennent d'individus de plus de vingt ans. Nous relativiserons l'importance à accorder à ces dents isolées indéterminées. En effet, il est probable qu'elles soient issues de mâchoires dont la classe d'âge a pu être avancée. Sur cent cinquante-sept jugales examinées, en place ou non dans la mâchoire, aucune déciduale n'est en revanche attestée. Les dents sont exclusivement des molaires M1, M2 et M3, et plus particulièrement des dernières et avant-dernières molaires (tabl. 3 - en annexe).

\section{2 - étude biométrique des molaires}

21 Rappelons que chez les éléphantidés le renouvellement dentaire se fait d'une manière tout à fait particulière. Le mammouth, comme l'éléphant aujourd'hui, possédait au total vingt-quatre dents jugales. Ainsi, par demi-mâchoire, six jugales se succédaient au cours de la vie de l'animal, soient trois déciduales (D2, D3, D4 encore appelées $\mathrm{m} 1, \mathrm{~m} 2$ ou $\mathrm{m} 3$ par certains auteurs) et trois molaires (M1, M2, M3). Le mode de remplacement des jugales est chez les éléphantidés tout à fait original puisqu'il se fait de manière dite « horizontale » suivant en fait une courbure bien spécifique. Ainsi, lorsqu'une jugale est en grande partie usée par phénomène d'abrasion, elle est poussée par la suivante et disparaît alors progressivement au profit de celle-ci, de plus grande taille et en tout début d'usure. Ainsi, il n'y a jamais plus de deux dents en fonction à la fois sur une demi-mâchoire, maxillaire ou hémi-mandibule. Cette succession ne vient à son terme que lorsque la dernière molaire définitive a fini, elle aussi, de s'user; l'animal, dans l'incapacité de se nourrir, meurt alors de faim, approximativement autour de 60 ans concernant les espèces actuelles (Laws 1966).

Les travaux auxquels nous nous sommes essentiellement référés pour déterminer la place des jugales de Mammuthus columbi dans la série dentaire sont ceux de H.F. Osborn (1942), de J.J. Saunders (1970) et de C.T. Madden (1981).

La biométrie de la molaire variant en fonction de son degré d'usure, il est impératif de prendre en considération ce dernier pour relativiser les mesures de longueur, largeur ou hauteur. Au cours de notre étude n'ont été retenues que les mesures effectuées à un stade d'usure permettant de conserver les dimensions maximales de la dent (dans l'idéal des stades d'usure A à C, fig. 8). Par longueur (Li) de la dent, nous entendons ici la longueur prise à la base de la couronne dentaire (Beden 1979). D'autres auteurs, tel que V.J. Maglio (1973) ou J.J. Saunders (1970) ont préféré mesurer la longueur (L) à mi- 
hauteur de la dent et perpendiculairement aux lames, d'autres encore parallèlement à la surface d'abrasion (Agenbroad in Agenbroad, Mead et Nelson 1994 - p. 183). La synthèse des mesures obtenues sur les molaires de Mammuthus columbi de Hot Springs est présentée tableau 5 .

Tableau 5 - Synthèse des mesures obtenues sur les molaires de Mammuthus columbi, Hot Springs.

\begin{tabular}{|l|rrrrrrrr|}
\cline { 2 - 9 } \multicolumn{1}{c}{} & $\mathrm{N}$ & $\mathrm{Li}$ & $\mathrm{l}$ & $\mathrm{H}$ & $\mathrm{h}$ & $\mathrm{e}$ & $\mathrm{F}$ lat. F occl. \\
\hline Nombre de M2 supérieures & & & & & & & & \\
ayant permis la mesure & 5 & 2 & 17 & 2 & 2 & 21 & 7 & 21 \\
Moyenne & & & 82 & & & 2,2 & 7,4 & 7,3 \\
Mini & 13 & 200 & 70 & 122 & 167 & 1,4 & 7 & 6 \\
Maxi & 16 & 220 & 93 & 130 & 174 & 2,8 & 8,25 & 9 \\
écart type & & & 6,98 & & & 0,38 & 0,45 & 0,8 \\
Coefficient de variation & & & 8,51 & & & 17,37 & 6,13 & 10,97 \\
\hline Nombre de M2 inférieures ayant & & & & & & & & \\
permis la mesure & 4 & 2 & 13 & & & 16 & 1 & 16 \\
Moyenne & 14 & 204,5 & 75,8 & & & 2,4 & & 6,9 \\
Mini & 12 & 194 & 70 & & & 1,9 & 8,25 & 5,25 \\
Maxi & 15 & 215 & 89 & & & 3,4 & 8,25 & 8,5 \\
écart type & & & 5,44 & & & 0,4 & & 0,8 \\
Coefficient de variation & & & 7,18 & & & 16,67 & 11,55 \\
\hline Nombre de M3 supérieures & & & & & & & & \\
ayant permis la mesure & 3 & 3 & 13 & 9 & 9 & 11 & 11 & 9 \\
Moyenne & & 338,3 & 96 & 192,6 & 192,4 & 2,3 & 6,8 & 7 \\
Mini & 18 & 300 & 70 & 130 & 144 & 1,6 & 4,5 & 5,75 \\
Maxi & 23 & 375 & 108 & 223 & 223 & 3 & 8,5 & 8 \\
écart type & & 37,53 & 9,9 & 27,22 & 23,97 & 0,49 & 1,09 & 0,85 \\
Coefficient de variation & & 11,09 & 10,31 & 14,13 & 12,46 & 21,57 & 16,07 & 12,12 \\
\hline Nombre de M3 inférieures ayant & & & & & & & & \\
permis la mesure & 1 & 2 & 10 & 1 & 1 & 9 & 2 & 10 \\
Moyenne & & & 86,5 & & & 2,5 & & 7 \\
Mini & & 300 & 72 & 200 & 206 & 1,8 & 4,25 & 5,25 \\
Maxi & 23 & $>300$ & 100 & 200 & 206 & 3,3 & 6 & 8,5 \\
écart type & & & 8 & & & 0,46 & 1,15 \\
Coefficient de variation & & & 9,25 & & & 18,65 & 16,41 \\
\hline
\end{tabular}

Légende : $\mathrm{N}$ : nombre de lames, $\mathrm{Li}:$ longueur de la couronne dentaire (en mm), 1 : largeur,

$\mathrm{H}$ : hauteur maximale, $\mathrm{h}$ : indice d'hypsodontie $\mathrm{h}=\mathrm{H} / 1 \times 100$, e : épaisseur d'émail,

$\mathrm{F}$ : fréquence laminaire (nombre de lames sur $10 \mathrm{~cm}$ ), mesure latérale et occlusale.

24 On notera que la position de certaines mâchoires dans le sédiment n'a pas permis d'effectuer la prise de mesures des molaires. C'est le cas de quatre crânes et de trois mandibules de la «partie est » et de la «partie sud-est» provenant tous d'individus adultes demeurés in situ sur le site (tabl. 4 - en annexe).

\section{3 - Attribution d'âges aux individus}

Une molaire, même si elle n'est pas entière, fournit nombre d'informations très utiles, comme l'espèce à laquelle appartient l'animal mais aussi son âge au moment de sa mort. Ainsi, l'étude de l'usure dentaire de chaque jugale nous informe sur l'âge au décès des individus. Cette analyse est par conséquent l'étape préalable à l'établissement de courbes de mortalité.

Une jugale d'éléphantidé est constituée par une série plus ou moins importante de lames transversales tenues les unes aux autres par le cément. Ces lames sont ellesmêmes formées par un fin repli d'ivoire recouvert d'une couche d'émail plus épaisse. Les lames s'usent quasi-perpendiculairement au fil de l'abrasion, ainsi leur taille diminue au fur et à mesure, ce qui conduit par conséquent à réduire la hauteur de la dent. Plusieurs méthodes ont été proposées dans le but d'apprécier le degré d'usure des jugales d'éléphantidés. Parmi elles, nous mentionnerons le rapport $\mathrm{Nf} / \mathrm{N}$ fréquemment utilisé (nombre de lames ouvertes/nombre total de lames). Toutefois, si cette formule 
est plus que convaincante en ce qui concerne les molaires entières, elle n'est pas applicable aux fragments dentaires.

La connaissance du stade d'usure de la dent est un paramètre très important car la seule position de la molaire dans la série jugale ne suffit pas à attribuer un âge à l'animal, ou alors celui-ci serait beaucoup trop approximatif, notamment s'agissant des dernières molaires.

Nous avons choisi d'opter pour la classification des stades d'usure de R. M. Laws (1966) obtenus sur les éléphants actuels, couplée à la méthode dont M. Beden (1979) a créé un modèle théorique applicable aux molaires inférieures « définitives » et dont nous avons de la même façon établi celui applicable aux molaires supérieures (fig. 8). Pour ce faire, près de deux cents jugales supérieures de mammouth provenant du gisement de Hanhoffen dans le Bas-Rhin ont été minutieusement analysées (Louguet-Lefebvre 2005, 2006). Les stades d'usure ainsi définis par M. Beden vont de la dent neuve (stade A) à la dent en fin de vie, abrasée à $100 \%$ (stade D4), en passant par les stades de destruction progressive des lames (stades D1 à D3 inclus). Cette méthode, comme celle de R.M. Laws, tient à la fois compte de la quantité relative de lames en fonction et de l'estimation des lames disparues par usure, mais elle tient également compte de l'angle formé par la table d'abrasion et la couronne dentaire. Ce dernier paramètre est d'autant plus utile dans le cas de molaires incomplètes. C'est pourquoi la corrélation entre cette méthode et celle de R.M. Laws est intéressante. Elle permet d'attribuer une classe d'âge relativement précise aux fragments de dents, notamment supérieures (tabl. 6).

Tableau 6 - Groupes d'âges attribués aux éléphantidés.

\begin{tabular}{|c|c|c|c|}
\hline \multicolumn{2}{|c|}{ R. M. Laws (1966) } & \multicolumn{2}{|c|}{ M. Beden (1979), S. Louguet-Lefebvre (2005) } \\
\hline Age (en années) & Stade d'usure dentaire & Type de dent & Stades d'usure dentaire \\
\hline \multirow{3}{*}{ jusqu'à 2} & \multirow{3}{*}{ I à IV } & D2 & Tous \\
\hline & & D3 & $\mathrm{A}$ à $\mathrm{C}$ \\
\hline & & D4 & $\mathrm{A}$ à B2 \\
\hline \multirow{3}{*}{3 à 4} & \multirow{3}{*}{ V à VII } & D3 & $\mathrm{D}$ \\
\hline & & D4 & $\mathrm{B} 3$ à $\mathrm{C}$ \\
\hline & & M1 & A \\
\hline \multirow[t]{2}{*}{6 à $10(+/-1)$} & \multirow[t]{2}{*}{ VII à IX } & D4 & C à D3 \\
\hline & & M1 & B1 à B3 \\
\hline \multirow{3}{*}{13 à $20(+/-2)$} & \multirow{3}{*}{$\mathrm{X}$ à XIII } & $\mathrm{D} 4$ & D3 à D4 \\
\hline & & M1 & B4 à D2 \\
\hline & & M2 & $\mathrm{A}$ à B2 \\
\hline \multirow{3}{*}{22 à $34(+/-2)$} & \multirow{3}{*}{ XIV à XX } & M1 & D3 à D4 \\
\hline & & M2 & B3 à D2 \\
\hline & & M3 & $\mathrm{A}$ à B2 \\
\hline \multirow[t]{2}{*}{36 à $47(+/-2)$} & \multirow[t]{2}{*}{ XXI à XXV } & M2 & $\overline{\mathrm{D} 3}$ à D4 \\
\hline & & M3 & $\mathrm{B} 3$ à $\mathrm{C}$ \\
\hline 49 à $62(+/-2)$ & XXVI à XXX & M3 & $\mathrm{D}$ \\
\hline
\end{tabular}

Les petites divergences existantes entre les âges attribués par différents auteurs peuvent impacter le profil de mortalité, c'est pourquoi il est capital de noter à quelle méthodologie nous faisons référence. Pour exemple, la classe XVI définie par R.M. Laws 
était attribuée à un âge proche de 26 ans, par la suite G.C. Craig lui fera correspondre un âge approximatif de 19 à 21 ans (Craig in Haynes 1991). C'est peut-être ce qui explique en partie les divergences observées entre les profils de mortalité de Hot Springs établis par différents auteurs, même si l'accroissement constant de l'échantillon étudié demeure un facteur indéniable de modification du profil. L'estimation de l'âge que nous proposons est ici basée sur le modèle de R.M. Laws établi sur les éléphants d'Afrique (1966), les éléphants actuels possédant une formule dentaire semblable à celle que possédait le mammouth et une espérance de vie que l'on suppose proche.

30 Le spécimen le plus complet (HS00140) présentait l'avantage de posséder ses deux mâchoires. L'analyse de la mandibule et des maxillaires nous a permis d'attribuer à l'individu un âge approximatif qui devait être proche de 40 à 45 ans. Ses dernières molaires inférieures présentent près de trois quarts de lames d'émail ouvertes et aucune lame disparue par l'usure, soit un stade d'usure B4 qui correspond au stade morphologique XXIII défini par R. M. Laws.

\section{5 - Résultats : élaboration d'un nouveau profil de mortalité}

\section{1 - Apport des études précédentes}

Plusieurs auteurs ont auparavant étudié les molaires de Mammuthus columbi de Hot Springs afin d'en dresser un profil de mortalité à un instant $\mathrm{T}$ de la fouille. Ainsi B. Dutrow en 1980 mène l'étude des quarante-six dents jugales mises au jour à cette date et détermine un NMI (nombre minimum d'individus) de huit (Dutrow 1980). En 1987, L.D. Agenbroad et J. Mead détermineront d'après les molaires pas moins de vingtsix individus (Agenbroad, Mead 1987) et quarante-trois d'après le décompte des défenses effectué en 1990, et cela pour seulement douze humérus (Agenbroad 1990). L'étude des quatre-vingt molaires permet alors à L.D. Agenbroad d'effectuer la révision de près de la moitié des âges qui avaient été attribués précédemment par B. Dutrow et de dresser un nouveau profil de mortalité, tandis que le NMI est alors estimé à cinquante-neuf individus (Dutrow in Agenbroad, Mead 1994). Toutefois, il s'agit d'un NMI de fréquence et non du NMI de combinaison auquel nous nous référons. Dans la mesure où sont prises en considération les dents isolées, le seul recours au NMI de fréquence a le désavantage de surestimer de manière considérable l'effectif des individus de moins de 40 ans. En effet, ces derniers ont la particularité de posséder deux dents en fonction par demi-mâchoire contre une dernière molaire unique au-delà de cet âge (la M3). Si l'on considère par exemple deux dents supérieures gauches isolées, soit une M1 présentant un stade d'usure $C$ et une M2 ayant atteint le stade d'usure A, il est possible que ces deux dents soient issues d'un même individu. Le profil obtenu alors présente ainsi une nette surreprésentation d'individus âgés de 10 à 19 ans et de 20 à 29 ans, puisque ces classes d'âge concentraient $80 \%$ de l'effectif, les $20 \%$ restant se répartissant quasi équitablement entre les dernières trois grandes classes d'âge. Tous les auteurs s'accordent en tous cas sur le fait qu'aucun petit de mammouth n'ait été mis au jour à Hot Springs, comme en témoigne l'absence de molaires déciduales. À en juger par le décompte des défenses effectué à ce jour par la fondation 
de L.D. Agenbroad, l'effectif de mammouths de Colomb pourrait atteindre cinquantesept individus en 2012 (http://www.mammothsite.com).

Figure 8 - Stades d'usure des molaires définitives d'Éléphantidés, d'après M. Beden 1979, pour la dentition inférieure, et $S$. Louguet-Lefebvre 2005, pour la dentition supérieure.

\begin{tabular}{|c|c|c|c|c|c|c|c|c|c|}
\hline \multirow{2}{*}{$\begin{array}{l}\text { Niveaux } \\
\text { d'usure }\end{array}$} & \multicolumn{4}{|c|}{$\begin{array}{l}\text { Augmentation des lames } \\
\text { en fonction }\end{array}$} & $\begin{array}{c}\text { Surface } \\
\text { d'abrasion }\end{array}$ & \multicolumn{4}{|c|}{$\begin{array}{c}\text { Destruction progressive } \\
\text { des lames }\end{array}$} \\
\hline & $B_{1}$ & $B_{2}$ & $\mathrm{~B}_{3}$ & $\mathrm{~B}_{4}$ & C & $D_{1}$ & $\mathrm{D}_{2}$ & $\mathrm{D}_{3}$ & $\mathrm{D}_{4}$ \\
\hline $\begin{array}{l}\text { Dent } \\
\text { supérieure }\end{array}$ & & & & & & 6 & 6 & 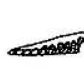 & $\infty$ \\
\hline $\begin{array}{c}\text { Dent } \\
\text { inférieure }\end{array}$ & 14 & In & & & & sing & 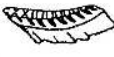 & 2 & 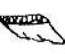 \\
\hline
\end{tabular}

\section{2 - Révision du profil de mortalité}

Les âges attribués à une dizaine de crânes par A. Lister en ayant recours aux mesures fournies par les condyles occipitaux (Lister in Agenbroad, Mead 1994) corroborent les âges que nous obtenons sur ces mêmes crânes par l'étude de leurs jugales. Par ailleurs, notre analyse permet de préciser les résultats issus des études ostéologiques menées par L.D. Agenbroad (1990) qui indiquaient une surreprésentation de mâles âgés de 10 à 35 ans.

33 Afin de déterminer le NMI (nombre minimum d'individus), nous avons évidemment pris en considération l'éventualité qu'une mâchoire supérieure (ou deux maxillaires), une mandibule (ou deux hémi-mandibules) et une ou plusieurs dents isolées puissent provenir d'un unique individu. A ce jour, seul le spécimen HS00140 atteste de ses deux mâchoires, d'ailleurs clairement associées par connexion anatomique au reste du squelette post-crânien, même si d'autres associations maxillaires-mandibule sont possibles. Pour déterminer le NMI nous avons tout d'abord totalisé le nombre maximal de dents de même nature (M3, M2...), de positions strictement identiques (supérieure ou inférieure, gauche ou droite) et de degré d'usure quasi similaire en admettant une marge d'appréciation d'un stade (par exemple B4 pour une dent et $C$ pour une autre). Nous avons ensuite réparti nos NMI par catégorie d'âge en nous référant au tableau 6 . Le NMI de combinaison a ensuite consisté en l'addition des NMI respectifs de chaque catégorie d'âges, soit trente-trois, auxquels nous avons ajouté trois, puisque trois mandibules non accessibles à cause de leur position dans le sédiment proviennent d'individus d'âge non connu (tabl. 7). Ce calcul nous a permis de revoir les dernières estimations à la baisse, puisque les jugales proviennent d'un minimum de trente-six individus. Cet effectif minimal est sans commune mesure avec celui fourni à ce jour par les défenses, qui rappelons-le atteindrait cinquantaine-sept. Les campagnes de fouilles à venir au cours des prochaines décennies devraient contribuer à amoindrir progressivement cet écart.

34 Notre révision du profil de mortalité de Mammuthus columbi, au vu des dernières données, indique que le piège a affecté une majorité de sujets adultes, puisque vingt- 
deux individus sur une totalité de trente-six identifiés d'après leurs molaires, sont décédés à un âge approximatif compris entre 25 et 38 ans (tabl. 7, fig. 9). L'absence de dents déciduales de même que l'absence de M1, non ou faiblement usée, confirment une absence de très jeunes individus et de juvéniles de moins de 10 ans. Le déficit en individus séniles associé au manque de très jeunes mammouths est surprenant.

Tableau 7 - Représentation du Mammuthus columbi de Hot Springs en NMIc (nombre minimum d'individus cumulé).

\begin{tabular}{|cc|}
\hline Classes d'âge (en années) & NMI c \\
\hline $0-12$ & 0 \\
$13-24$ & 4 \\
$25-38$ & 22 \\
$39-47$ & 3 \\
$>47$ & 4 \\
Total (d'âge connu) & 33 \\
Âge non connu & 3 \\
\hline TOTAL & 36 \\
\hline
\end{tabular}

Figure 9 - Profil de mortalité de Mammuthus columbi, Hot Springs, Dakota du Sud ( \pm 1 an pour la classe 6-10 ans et \pm 2 ans pour les classes supérieures; NMlc : 36 déterminés à partir des molaires).

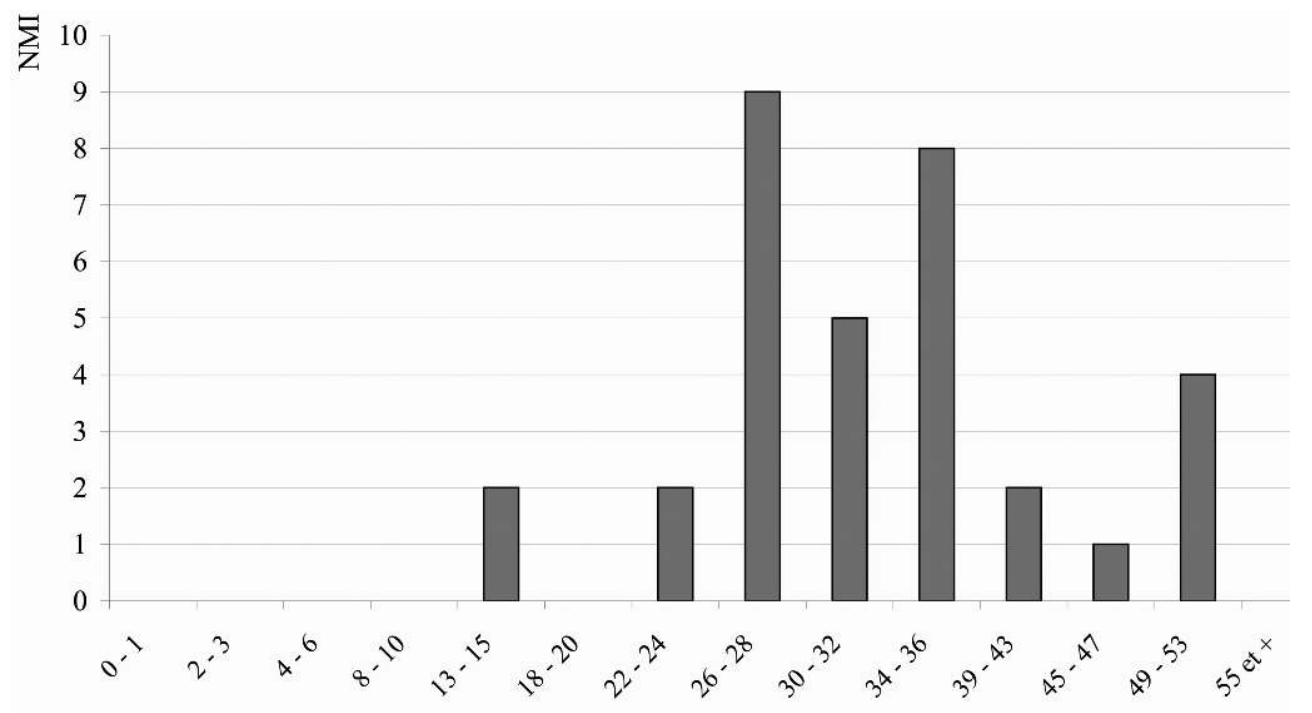

âge approximatif (en années) 


\section{6 - Discussion}

\section{1 - Caractérisation des différents profils de mortalité}

35 Les mammouths, à l'image des éléphants, devaient être des animaux grégaires. Chez les éléphants actuels, le troupeau, conduit par une matriarche peut contenir jusqu'à une trentaine d'individus d'une même famille, principalement des femelles accompagnées de leurs petits, des jeunes et un mâle âgé. Les mâles ayant atteint la maturité sexuelle depuis deux ou trois années sont quant à eux exclus du troupeau et adoptent alors une vie solitaire pour ensuite se regrouper entre eux, ne retournant voir les femelles que pour la procréation (Laws 1966).

En Amérique du Nord, la plupart des assemblages de mammouths présentent un profil de type $B$ résultant d'une mortalité sélective ayant affecté un troupeau mixte (cas des sites à pointes de Clovis, fig. 1), ou de type $\mathrm{C}$ résultant d'une mortalité sélective n'ayant affecté que des mâles ou de mortalité non-sélective ayant affecté une population en déclin (Haynes 1991). Le type $C$ est par exemple rencontré sur le site paléontologique de Lamb Spring dans le Colorado où une quarantaine de mammouths de Colomb a été mise au jour (Stanford et al. 1981).

La mortalité de masse ayant affecté une population mixte, stable et en pleine expansion (de type A) a la spécificité de comprendre essentiellement des individus très jeunes ou juvéniles (près de $50 \%$ de moins de 12 ans) ainsi que des femelles. Quant aux autres classes d'âge du type $\mathrm{A}$, leur effectif décroît au fur et à mesure de l'augmentation de l'âge (Haynes 1987). Ce profil traduit le plus souvent une mort catastrophique pouvant résulter d'une épidémie, d'une inondation, d'une trop forte sécheresse, d'un incendie.

Le profil de type $B$, caractérise lui un nombre extrêmement important de très jeunes et de juvéniles (plus des deux tiers ont moins de 12 ans). Quelques jeunes adultes d'approximativement 13-24 ans ainsi que quelques individus en fin de vie peuvent également y être représentés. Néanmoins, les adultes et les adultes âgés, d'environ 22 à 48 ans, plus à même de déjouer les assauts de prédateurs ou les pièges tendus par les hommes, ne sont quasiment pas présents.

Quant au profil de type $\mathrm{C}$, il résulterait d'une mortalité non-sélective sur une population en déclin ou d'une mortalité sélective n'ayant affecté que des mâles. Les principales victimes y sont des adultes d'environ 25-38 ans, suivis de jeunes adultes d'approximativement 13-24 ans ainsi que d'adultes âgés de près de 37-48 ans. Les très jeunes individus et les juvéniles de moins de 12 ans, comme les plus anciens de plus de 49 ans, sont par conséquent sous-représentés. Le profil de type $C$, pouvant correspondre à une mortalité catastrophique survenue sur une population en déclin présente un minimum d'individus subadultes.

Le profil de mortalité obtenu à Hot Springs ne correspond de façon précise à aucun des principaux types observés par G. Haynes (1987).

\section{2 - Le piège aurait ponctuellement affecté des mâles isolés ou en petits groupes}

L'interprétation première, avancée par le docteur L.D. Agenbroad et son équipe, est que le piège aurait agi sur des mâles solitaires ou sur des couples de mâles mais n'aurait concerné aucune famille composée de femelles accompagnées de leurs petits. En effet, 
le manque de déciduales (D2, D3 ou D4) confirmant l'absence de jeunes individus pourrait suggérer que le piège n'ait pas touché de groupes familiaux.

Ce profil de mortalité ne trouve que très peu d'équivalents, tant du point de vue paléontologique que du point de vue éthologique. Cependant, grâce au concours de Gary Haynes il nous a été possible de faire le parallèle entre le profil obtenu à Hot Springs et l'estimation des proportions de mâles qu'il a faite à partir de populations de Loxodonta mâles issues de différents parcs nationaux du nord-ouest du Zimbabwe (communications personnelles de 2005 et 2008, Haynes 1991). Bien que son estimation des âges se soit faite de façon approximative, il ressort de ses nombreuses observations une nette surreprésentaion, chez les mâles, d'éléphants en pleine force de l'âge. Les 13-36 ans y totalisent largement plus de la moitié de l'effectif, suivis dans une bien moindre mesure par les plus de 37 ans accompagnés d'une absence d'individus de moins de 12 ans. Ces estimations en proportions d'éléphants mâles avancées par Gary Haynes ne prennent ici en considération que les mâles qui n'étaient pas intégrés aux troupeaux mixtes. Il se peut qu'elles sous-estiment quelque peu les individus de plus de 37 ans si l'on considère que des mâles adultes d'approximativement 37 à 48 ans peuvent temporairement être associés aux troupeaux mixtes même s'ils demeurent principalement composés de femelles et de jeunes (Haynes 1991).

La surreprésentation de mammouths adultes âgés de 25 à 38 ans, soit près de deux individus sur trois dans le cas de Hot Springs, la faible proportion des autres classes d'âges et l'absence d'individus de moins de 12 ans sont autant de points communs entre Hot Springs et les sites mentionnés ci-dessus. L'interprétation d'un tel profil de mortalité demeure difficile à interpréter, d'autant qu'une telle configuration n'est pas suffisamment documentée à l'heure actuelle, comme nous venons de le mentionner. Toutefois, les fortes similarités observées entre le profil de Hot Springs et les proportions de mâles observées au Zimbabwe tendraient à rapprocher ce profil du type $C$ défini par Gary Haynes, résultant notamment d'une mortalité sélective n'ayant affecté que des mâles, à ceci près que dans les deux cas les subadultes sont absents.

43 Si l'on excepte le site de Hot Springs, qui fait figure d'exception, la plupart des dépôts à forte accumulation osseuse d'éléphantidés (fossiles ou contemporains) résultent ordinairement principalement de troupeaux mixtes ne contenant au plus que quelques mâles adultes (Haynes 1991).

\section{3 - Le piège aurait ponctuellement affecté des mâles et troupeaux mixtes}

Si l'on se fie aux éléphants d'Asie, les éléphantidés sont d'excellents nageurs, les adultes n'hésitant pas à se mettre en danger pour porter assistance à leur descendance. Il n'est donc pas dénué de sens d'imaginer que des femelles adultes aient pu à Hot Springs porter secours aux plus jeunes, plus insouciants et vulnérables, en les aidant à s'extraire du plan d'eau. Le fait qu'il n'y ait pas de jeunes ne signifie donc pas forcément que le piège n'ait affecté que les mâles isolés, il peut également s'agir de membres issus de groupes de femelles dont les plus jeunes, moins lourds, auraient pu réchapper.

Cette hypothèse pourrait éventuellement être soutenue par la surreprésentation, en nombre de restes comme en NMI, de pachydermes de près de cinq à dix tonnes au détriment des mammifères de moindre taille (ours géant à face courte de près de 600 $\mathrm{kg}$, camélidé, lama, antilope d'Amérique, loup, coyote, blaireau..., Agenbroad, Mead 
1994) et de jeunes mammouths encore loin de leur maximum pondéral. En effet, si l'on en juge par nos éléphants actuels, le poids du petit mammouth à la naissance devait être proche de $100 \mathrm{~kg}$, puis augmentait progressivement, à raison d'un kilo par jour, jusqu'à atteindre un poids d'adulte avoisinant les huit tonnes. Ainsi, il apparaît que les éléphantidés pris au piège à Hot Springs ne devaient guère peser moins de 4,5 tonnes.

L'étude isotopique de l'oxygène menée par D.C. Fisher sur des échantillons de défenses provenant de Hot Springs semble relever d'une distribution annuelle «bimodale » de la mortalité. Le piège pourrait avoir fonctionné plus particulièrement au printemps et en automne (Fisher et al. 2003). Cette répartition de la mortalité pourrait ainsi s'expliquer par l'état particulièrement instable des berges détrempées en ces deux saisons, tandis que le sol asséché en été et gelé en hiver devaient faciliter la remontée des pachydermes via des berges plus stables ne se dérobant pas sous leur poids colossal.

Enfin, même si trop peu d'éléments osseux ont permis d'établir le sex-ratio de la population, les femelles ne sont pas pour autant écartées de cette accumulation comme en témoigne l'un des plus jolis spécimens en connexion in situ (squelette post-crânien 13S23W) attribué par A. Lister à une femelle (Lister in Agenbroad, Mead 1994). Cela remet en cause l'hypothèse évoquée précédemment qui évoque une possible absence de groupes familiaux dans la composition de l'assemblage osseux de proboscidiens.

\section{7 - Conclusion}

La contribution de l'analyse des éléphantidés de Hot Springs est incontournable puisqu'elle complète de façon inattendue le référentiel de mortalité naturelle déjà existant. Toutefois, il nous est difficile d'interpréter avec exactitude le profil de mortalité de Mammuthus columbi obtenu. En effet, est attestée une surreprésentation notable des adultes et l'absence de jeunes animaux parmi un minimum de trente-six individus, compte tenu de l'analyse des molaires. Ce type de configuration ne connaît que peu d'équivalent et demeure non suffisamment documenté à l'heure actuelle. Par conséquent, aucune interprétation n'est à privilégier. Le piège aurait pu affecter de façon ponctuelle des mâles isolés, des petits groupes de mâles, ou encore des troupeaux mixtes dont les plus jeunes, moins lourds, auraient su s'extirper avec l'aide des adultes. A l'inverse, il est envisageable que les berges instables aient cédé sous le poids colossal des pachydermes adultes, ne leur laissant que peu de chance de s'extraire de ce bourbier qui n'offrait alors plus aucune prise en cas de chute, autrement dit aucun échappatoire.

Les fouilles estivales menées par L.D. Agenbroad et son équipe pourraient permettre de réactualiser le NMI au gré des dernières découvertes et de préciser le profil de mortalité d'ici quelques années. 


\section{BIBLIOGRAPHIE}

AGENBROAD L.D. 1989 - Spiral Fractured Mammoth Bone from Nonhuman Taphonomic Processes at Hot Spring Mammoth Site. In : R. Bonnichsen, M.A. Sorg (Eds.), Bone Modification. Orono : Center for the Study of the First Americans, University of Maine, p. 139-147.

AGENBROAD L.D. 1990 - The mammoth population of the Hot Springs site and associated megafauna. In : L.D. Agenbroad, J. I. Mead, L. Nelson (Eds.), Megafauna and Man. The discovery of America's Heartland. Hot Springs, South Dakota : Mammoth Site of Hot Springs, p. 32-35.

AGENBROAD L.D., MEAD J.I. 1987 - Age structure analysis of Mammuthus columbi, Hot Springs, South Dakota, Current Research in the Pleistocene 4, p. 101-102.

AGENBROAD L.D., MEAD J.I. 1994 (Eds.) - The Hot Springs Mammoth Site : a Decade of Field and Laboratory Research in Paleontology, Geology, and Paleoecology, Mammoth Site of Hot Springs. South Dakota, Inc., Hot Springs. 451 p.

ANDREWS P., LORD J.M., NESBIT-EVANS E.M. 1979 - Patterns of ecological diversity in fossil and mammalian faunas. Biology journal Linnean Society, 11, p. 177-205.

AULAGNIER S., HAFFNER P., MITCHELL-JONES T., MOUTOU F., ZIMA J. 2010 - Guide des mammifères d'Europe, d'Afrique du Nord et du Moyen-Orient. Paris, Ed. Delachaux et Niestlé. 272p.

BEDEN M. 1979 - Les Éléphants d'Afrique Orientale : systématique, phylogénie, intérêt biochronologique. Thèse de la Faculté des Sciences Universitaires de Poitiers, 2 vol. , 567 p. (inédit).

CRAIG G.C. 1991 - in : Haynes G. 1991 - Mammoths, mastodonts and elephants. Biology, behavior, and the fossil record. Cambridge University Press, $413 \mathrm{p}$.

DUTROW B. 1980 - Metric analysis of a Late Pleistocene Mammoth Assemblage, Hot Springs, South Dakota. M.S. thesis, Southern Methodist University (unpublished).

FIGUEIRIDO B., PÉREZ-CLAROS J. A., TORREGROSA V., MARTÍN-SERRA A., PALMQVIST P. 2010 Demythologizing Arctodus simus, the "Short-Faced" long-legged and predaceous bear that never was, Journal of Vertebrate Paleontology, 30 : 1, p. 262-275.

FISHER D.C, FOX D.L., AGENBROAD L.D. 2003 - Tusk growth rate and season of death of Mammuthus columbi from Hot Springs. South Dakota, USA Deinsea, 9, p. 117-133.

FLEMING T.H. 1973 - Numbers of mammal species in north and central America forest communities. Ecology, 54, $\mathrm{n}^{\circ}$ 3, p. 555-563.

GUÉRIN C., FAURE M. 1987 - Grands Mammifères. In :J.C. Miskovsky et al., Géologie de la Préhistoire : méthodes, techniques, applications, Géopré édit., Maison de la Géologie, Paris, p. 801-830.

HANNUS L.A. 1989 - Flaked Mammoth Bone from the Lange Ferguson Site, With River Badlands Area, South Dakota. In : R. Bonnichsen, M.A. Sorg (Eds.), Bone Modification. Orono : Center for the Study of the First Americans, University of Maine, p. 395-412.

HAYNES G., 1987 : Proboscidean die-offs and die-outs : Age profiles in fossil collections. Journal of Archaeological Sciences. 14(6) : p. 659-668. 
HAYNES G. 1991 - Mammoths, mastodonts and elephants. Biology, behavior, and the fossil record. Cambridge University Press, $413 \mathrm{p}$.

LAURY R.L. 1990 - Geologic History of the Mammoth site and Surrounding Region Hot Springs Area, Fall River and Custer Counties, South Dakota : An Overview. In : L.D. Agenbroad, J.I. Mead, L.W. Nelson (Eds.), Megafauna and Man. Flagstaff, Arizona, p. 15-21.

LAWS R.M. 1966 - Age criteria for the African elephant, Loxodonta a. africana. East African Wildlife Journal. 4, p. 1-37.

LEGENDRE S. 1989 - Les communautés de mammifères du Paléogène (Éocène supérieur et Oligocène) d'Europe occidentale : structures, milieux et évolution. Ed. F. Pfeil., München 265 p.

LOUGUET-LEFEBVRE S. 2005 - Les mégaherbivores au Paléolithique moyen en Europe du NordOuest : paléoécologie, taphonomie et aspects palethnographiques. British Archaeologial Reports, International Series / S1451, 357 p.

LOUGUET-LEFEBVRE S. 2006 - Determining the age of death of Proboscids and Rhinocerids from dental attrition. Oxbow, Icaz Durham 2002, International Council of Archaeozoology, 9th Conference, 23-28 August, p. 179-188.

MADDEN C.T. 1981 - Mammoths of North America. Ph. D. thesis of Colorado University, Ann Arbor, 271 p. (unpublished).

MAGLIO V.J. 1973 - The origin and evolution of the Elephantidae. The American Philosophical Society, $62,149 \mathrm{p}$.

MEAD J., HELVY R.H., AGENBROAD L.D., 1990 - Late Pleistocene Invertebrates and Plant Remains, Mammoth Site, Black Hills, South Dakota. In : L.D. Agenbroad, J.L. Mead, L. Nelson (Eds.), Megafauna and Man. The discovery of America's Heartland. Hot Springs, South Dakota : Mammoth Site of Hot Springs, p. 9.

MONTUIRE S., MARCOLINI F. 2002 - Palaeoenvironmental significance of the mammalian faunas of Italy since the Pliocene. Journal of Quaternary Science, vol. 17(1), p. 87-96.

OSBORN H.F. 1942 - Proboscidea, a Monograph of the Diversity, Evolution, Migration and Extinction of the Mastodons and Elephants of the World, vol. II : Stegodontoidea, Elephantoidea. American Museum of Natural History, New York, p. 805-1675.

PÉAN, S. 2001 - Comportements de subsistance au Gravettien en Europe centrale. Thèse Doct. MNHN Paris. 413 p. (unpublished).

SAUNDERS J.J. 1970 - The distribution and taxonomy of Mammuthus in Arizona. M.Sc. thesis of Arizona University, Tucson, 115 p. (unpublished).

STANFORD D., WEDEL W. R., SCOTT G. R. 1981 - Archaeological investigations of the Lamb Spring site. Southwestern Lore 47(1), p. 14-27.

VALVERDE J.A. 1964 - Remarque sur la structure et l'évolution des communautés de vertébrés terrestres. Terre et Vie, III, p. 121-154.

\section{ANNEXES}

ANNEXE 
Tableau 3 - Données obtenues sur les molaires de Mammuthus columbi, Hot Springs.

\begin{tabular}{|c|c|c|c|c|c|c|c|c|c|c|c|c|c|c|c|}
\hline $\mathrm{N}^{o}$ pièce & Photo Position dent & $\mathrm{N}$ & $\mathrm{Nd}$ & $\mathrm{Nf}$ & $\mathrm{Li}$ & 1 & $\mathrm{H}$ & $\mathrm{h}$ & u & $\mathrm{e}$ & $\mathrm{F}$ lat. & Foccl. 1 & Laws & Age approx. & Remarques \\
\hline Sans $N^{0} 17$ & M3 sup. & $?$ & ?" & $?$ & & & & & $?$ & & & & & & Accessibilité gẽnée dans le maxillaire \\
\hline MSL692 & M2 sup. g. & 15 & $-15 x$ & $11-$ & & & & & c & 1,8 & 7 & 7 & XVI-XVII & $25-38$ & Dent casscéc. Présence d' $1 / 2$ lame \\
\hline MSL739 & M2 sup. g. & 14 & $-14 x$ & 6 & & 78 & & & B3 & 1,7 & 8,25 & 8 & $\mathrm{xIV}$ & 13-24 & \\
\hline MSL157 & M2 sup. g. & 10 & $-10\left(x_{2}\right)$ & 10 & & 90 & & & $\mathrm{c}$ & 1.4 & 7.5 & 7.5 & XVI-XVII & $25-38$ & Cèment important \\
\hline MSL 734 & M3 sup. g. & 8 & $x 8$ - & 0 & & 99 & 213 & 215 & A & & 6,25 & & XVII-XVIII & $25-38$ & \\
\hline MSL.737 & M2/3 sup. d. & 7 & -7. & 7 & & 92 & & & c & 1,9 & 5,5 & 5,5 & & & \\
\hline MSL738 & M3 sup. d. & 9 & $x^{9}-$ & 0 & & 100 & 190 & 190 & A & & & & XVII-XVIII & $25-38$ & Dans sa malrice osseuse \\
\hline MSL735 & M1:2 sup. g. & 7 & $\mathrm{x} 7$ & 0 & & 82 & & & A & & 6,5 & & & & \\
\hline MSL736 & M sup. g. & 4 & $x 4-$ & 0 & & (80) & & & $\mathrm{A}$ & & & & $?$ & ? & \\
\hline MSL239 & M sup. & 3 & $-3-$ & 0 & & & & & $?$ & & & & $?$ & ? & \\
\hline MSL119 & $\mathrm{M} 2: 3$ & 1 & -1 & 0 & & (91) & & & ? & & & & ? & ? & \\
\hline MSL.120 & $\mathrm{M} 2 / 3$ sup. & 4 & $-4-$ & 0 & & (88) & & & $?$ & & & & ? & ? & \\
\hline MSL519 & M inf. d. & 6 & -6 & 0 & & & & & $?$ & & & & ? & ? & \\
\hline MSL741 & M2/3 sup. g. & 9 & -9 & 9 & & (76) & & & D1 & 1,7 & 7,5 & 7,5 & $?$ & ? & \\
\hline MSL. 742 & M3 sup. d. & 9 & $\times 9-$ & 7 & & 98 & 211 & 215 & 133 & 1,6 & 8.5 & 8 & $x \mathrm{x} I$ & $25-38$ & \\
\hline MSL743 & M3 sup. $\mathrm{g}$. & 16 & $x 16-$ & 6 & & 100 & 223 & 223 & B2 & 1,6 & 8 & 8 & $\mathrm{xx}$ & $25-38$ & \\
\hline MSL745 & M3 inf. d. & 8 & $\infty 8(x)$ & 8 & & (94) & & & $\mathrm{D} 2$ & 2,6 & & 5,25 & XXVII & $>47$ & \\
\hline MSL746 & M3 sup. d. & 12 & 12 & 6 & & 108 & 191 & 177 & B3.3. & 2 & 6.25 & 7 & $\mathrm{xXI}$ & $25-38$ & \\
\hline MSL749 & M3 sup. y. & 5 & $-5 x$ & 2 & & & & & D2 & 3 & 4,5 & & XXVII & $>47$ & Morphologic archaïque \\
\hline MSL 747 & M2 sup. d. & 9 & $x 9(\infty)$ & 9 & & 88 & & & D1 & 1,6 & 7,5 & 8,5 & XVIII & $25-38$ & \\
\hline MSL.748 & M sup. $g$. & 5 & $-5 x$ & 0 & & & & & & & 8 & & $?$ & $"$ & \\
\hline MSL750 & M inf. ? & 9 & $-9-$ & 0 & & & & & & & 9 & & ? & ? & \\
\hline MSL812 & M3 inf. d. & 13 & $\times 13-$ & 5 & & 97 & 200 & 206 & 132 & 1,8 & 6 & 8 & $x x$ & $25-38$ & \\
\hline MSL1083 & M sup. & 3 & $-3-$ & 0 & & & & & & 2.1 & 6 & & $?$ & $?$ & \\
\hline MSLL1139 & M2 sup. g. & 9 & $\infty 9(x)$ & 9 & & (92) & & & D3 & 1,8 & 7,5 & 7,5 & XXI & $25-38$ & \\
\hline MSL.531 & M sup. & 2 & $-2-$ & 0 & & & $(16(1))$ & & & 1,4 & & & $?$ & $?$ & \\
\hline MSL575 & $?$ & 1 & $-1-$ & 0 & & & & & & 2 & & & $?$ & $?$ & Fragment de lame \\
\hline MSL576 & ? & 1 & -1 & 0 & & & & & & 1,7 & & & $?$ & ? & Fragment de lame \\
\hline MSL.1196 & ? & 1 & -1 & $?$ & & & & & & & & & $?$ & $?$ & Fragment de lame \\
\hline MSLI197 & ? & 1 & $-1-$ & 0 & & & & & & & & & $?$ & ? & \\
\hline MSL521 & ? & 1 & -1 & 1 & & & & & & 1,8 & & & ? & ? & \\
\hline HSMS85001 & $\mathrm{M} 1 / 2$ & 7 & $x 7(x)$ & 7 & & (69) & & & D3 & & 9 & 9 & & & \\
\hline HSMS 85004 & M2 sup. d. & 15 & (ळ) $15 x$ & 13 & 220 & 92 & & & c & 2,3 & 7 & 7,25 & XVI-XVII & $25-38$ & \\
\hline HSMS76391 & M3 sup. g. & 10 & $\infty 10 \mathrm{x}$ & 10 & & (86) & & & D2 & 2 & 6,75 & 6 & XXVII & $>47$ & \\
\hline MSL 727 & MI sup. g.? & 8 & $\approx 8-$ & 8 & & 73 & & & CD1 & 1.5 & 9 & 8 & XI-XII & $13-24$ & Doute M. primigenaus (Lister, 1994) \\
\hline
\end{tabular}

Lègende : $\mathrm{M}$ : molaire (inf./ sup. / d. / g.: infëricure / supérieure / droite / gauche) ; $\mathrm{N}$ : nombre de lames ; Nf: nombre de lames fonctionnelles

Nd : formule laminaire ; + : des lames sont inaccessibles ; $\infty$ : lames disparu par usure horizontale ; $(x)$ : lames disparu par contact entre 2 dents ; - : cassure ; $\mathrm{x}$ : lamelle. $\mathrm{Li}:$ longueur de la couronne dentaire (en mm), I: largeur, $\mathrm{H}$ : hauteur maximale, $\mathrm{h}:$ indice d'hypsodontie $\mathrm{h}-\mathrm{H} / \mathrm{I} \times 100$. u : stade d'usure, : : épaisseur d'ćmail, F: frèquence lamin
Laws : Groupes d'âge définis par Laws. Âge en années

\begin{tabular}{|c|c|c|c|c|c|c|c|c|c|c|c|c|c|c|c|}
\hline$N^{N o p i e c e c ~}$ & Photo Position dent & $\mathrm{N}$ & $\mathrm{Nd}$ & $\mathrm{Nf}$ & $\mathrm{Li}$ & 1 & $\mathrm{H}$ & h & u & $\mathrm{s}$ & F lat. & F occl. & Laws & Agc approx. & Remarques \\
\hline \multirow[t]{4}{*}{$\frac{7.157}{78.157}$} & M2 sup. d. & 9 & $\propto 9(x)$ & 9 & & (77) & & & D2 & 2,3 & & 6 & $\mathrm{XX}$ & $25-38$ & \\
\hline & M3 sup. d. & $>18$ & 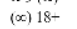 & 5 & 340 & 90 & $(130)$ & 144 & $\mathrm{~B} 2$ & 2,5 & 7 & 7 & & & \\
\hline & M2 sup. g. & 7 & $\propto 7(\infty)$ & 7 & & $(80)$ & & & D2 & 2,3 & & 6 & & & \\
\hline & M3 sup. $\mathrm{g}$. & $>18$ & (ळ) $18^{-}$ & 5 & 330 & 90 & & & $\mathrm{~B} 2$ & 2,5 & 7 & 7 & & & \\
\hline \multirow{4}{*}{ MSL1172 } & M2 inf. g. & 14 & -14 & 12 & & 87 & & & c & 3,2 & & 5,5 & XVI-XVII & $25-38$ & Cément important \\
\hline & M3 inf, g. & $?$ & $?$ & 0 & & & & & $\mathrm{~A}$ & & 4,25 & & & & \\
\hline & M2 inf. d. & 10 & -10 & 9 & & 89 & & & c & 3,2 & & 4.75 & & & \\
\hline & M3 inf. d. & & ? & 0 & & & & & A & & 4,25 & & & & \\
\hline \multirow[t]{2}{*}{ Sans $\mathrm{N}^{*} 9$} & M2 inf. $g$. & 13 & -13 & 12 & & 72 & & & c & 2,4 & & 7 & XVI-XVII & $25-38$ & \\
\hline & M3 inf. g. & & & 0 & & & & & & & & & & & \\
\hline \multirow{2}{*}{ Sans $\mathrm{N}^{*} 10$} & M3 inf. d. & $\gg 17$ & $\propto 17+$ & 13 & (290) & 97 & & & B4 & 2,9 & & 5.5 & XXIII & $39-47$ & \\
\hline & M3 inf. g. & $\geqslant 17$ & $\propto 17+$ & 13 & (290) & 100 & & & B4 & 2,9 & & 5.5 & & & \\
\hline \multirow{4}{*}{$87.523 / \div 108$} & $\mathrm{M} 2$ inf, 0 & 6 & $\propto 6(\infty)$ & 6 & & & & & $\mathrm{D}^{3}$ & 34 & & 6 & $\mathrm{XXI}$ & $25-38$ & Cément dcs M3 important \\
\hline & M3 inf. g. & 22 & $(\infty) 22$. & 8 & $(300)$ & 80 & & & B3 & 3,3 & & 7,5 & & & \\
\hline & M2 inf. d. & 6 & $\infty 6(\infty)$ & 6 & & & & & D3 & 3,4 & & 6 & & & \\
\hline & M3 inf. d. & 22 & (ळ) 22 . & 9 & $(300)$ & 80 & & & B3 & $\begin{array}{l}3,4 \\
3,3\end{array}$ & & 7,5 & & & \\
\hline \multirow[t]{4}{*}{ Sans $\mathrm{N}^{2} 11$} & M2 inf. d. & 10 & $\propto 10$ & 10 & (203) & 73 & & & D1 & 2,5 & & 6 & XVIII & $25-38$ & Cément des M2 important \\
\hline & M2 inf. g. & 10 & $\propto 10$ & 10 & & 76 & & & D1 & 2,5 & & 6 & & & \\
\hline & M3 inf. d. & $?$ & ? & 0 & & & & & $\mathrm{~A}$ & & & & & & \\
\hline & M3 inf. g. & $?$ & ? & 0 & & & & & A & & & & & & \\
\hline \multirow{2}{*}{ MSL 744/85.002 } & M2 sup. g. & 13 & $-13 x$ & 13 & & 79 & & & c & 2,8 & & 7,5 & XVI-XVII & $25-38$ & Cément de la M2 important \\
\hline & M3 sup. g. & 4 & 4. & 0 & & & & & & & & & & & \\
\hline \multirow{3}{*}{ HS00035 / MSL937 } & M1 inf. d. & 2 & $\infty 2(\infty)$ & 2 & & $(70)$ & & & D4 & & & & XVI & $25-38$ & Cément des M2 important \\
\hline & M2 inf. d. $>>$ l & $12 ?$ & (ळ) $12 ? \mathrm{x}$ & 11 & & 70 & & & B4 & 2,4 & & 8 & & & \\
\hline & M2 inf. g. & $13 ?$ & (œ) $13 ? \mathrm{x}$ & 13 & (194) & 72 & & & B4 & 2,4 & & 7,75 & & & \\
\hline \multirow[t]{2}{*}{ Sans $\mathrm{N}^{2} 12$} & M2 inf. d. & 12 & $12 \mathrm{x}$ & 12 & & 74 & & & c & 1,86 & & 675 & XVI-XVII & $25-38$ & \\
\hline & M3 inf. d. & $?$ & ? & & & & & & $\mathrm{A}$ & & & & & & \\
\hline \multirow[t]{3}{*}{ " Manfred " } & M2 sup. d. & 13 & $\infty 13 x$ & 12 & (206) & 80 & & & c & 2,4 & & 7,25 & XVI-XVII & $25-38$ & \\
\hline & M2 sup. g. & 13 & $\infty 13 x$ & 12 & (213) & 84 & & & c & 2,4 & & 7 & & & \\
\hline & M3 sup. g. & $?$ & $?$ & 0 & & & & & $\mathrm{~A}$ & & & & & & \\
\hline Sans $N^{*} 13$ & M2 inf. d. & 13 & $\infty 13(\infty)$ & 13 & & 76 & & & D1 & 2,3 & & 6,5 & xVIII & $25-38$ & \\
\hline HSO0297 & M sup. d. & 5 & -5. & 0 & & (72) & $(178)$ & 247 & $?$ & $?$ & 7 & & $?$ & $?$ & \\
\hline Sans $\mathrm{N}^{\mathrm{s}} 14$ & $\mathrm{M} 2 / 3$ sup. & $>11$ & $\infty 111$ ? & 11 & & $(\$ 2)$ & & & D & 2,6 & 7 & & & & Encore enfouie dans le sédiment \\
\hline HSO02I & M sup. g. & $>6$ & 6+ & 6it & & (77) & & & $?$ & ? & & 7 & $?$ & ? & Coincée entre maxillaire et sédiment \\
\hline 05 IIS105 & M sup.? & 3 & $\times 3$ & 0 & & (75) & (158) & 211 & $\mathrm{~A}$ & & & & $\because$ & ? & \\
\hline HIS00706? & M2 sup. d. & 13 & $\infty 13$ & 13 & & 80 & & & c & 2,6 & & 7,5 & 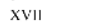 & $25-38$ & Seule en place dans le maxillaire \\
\hline Sans $\mathrm{N}^{\circ} 19$ & M3 sup. g. & 14 & 14. & 6 & (230) & $(102-)$ & 190 & $186+$ & 132 & 1,88 & 6,5 & 8 & $x x$ & $25-38$ & \\
\hline HS004118 / HSH H & M2 sup. g. & $>11$ & $\approx 11+$ & 11 & & 80 & & & D1 & 2,6 & & 6 & $x \mathrm{VIII}^{\prime}$ & $25-38$ & Seule en place dans le maxillaire \\
\hline $90 \mathrm{HLO12}$ & M2/3 sup.? & 9 & $\infty 9$ & 9 & & (70) & & & D3 & 2,2 & & 7 & $?$ & ? & En partie masquée par le sédiment \\
\hline Sans $\mathrm{N}^{\mathrm{x}} 15$ & $\mathrm{M} 2 / 3$ inf. $\mathrm{g}$. & $>11$ & $11+$ & $11+$ & & & & & B4 & 2,8 & & 6 & & & Ilémi-mandibule sortant du sédiment \\
\hline $\operatorname{Sans} \mathrm{N}^{*} 16$ & M3 inf d. & 10 & $\infty 10$ & 10 & & ${ }_{84}$ & & & $\mathrm{DI}$ & 2,3 & & 6.5 & $\mathrm{xxy1}$ & $>47$ & Dans l'hémi-mandibule. Cément + \\
\hline
\end{tabular}




\begin{tabular}{|c|c|c|c|c|c|c|c|c|c|c|c|c|c|c|c|}
\hline$V$ pièce & Phote Pasition dent & $\mathrm{N}$ & $\mathrm{Nd}$ & $\mathrm{Nr}$ & Li & 1 & II & $\mathrm{h}$ & u & $\mathrm{e}$ & F lat. & F occl. & Laws & Age approx. & Remarques \\
\hline \multirow[t]{4}{*}{$89 \mathrm{HS} 062$} & M2 sup. g. & 11 & $\infty, 11$ & 11 & & 70 & & & $\mathrm{D} 2$ & 2,3 & & 7,5 & $\mathrm{xX}$ & $25-38$ & Cément des M3 important \\
\hline & M3 sup. g. & $>3$ & $(\infty) 3^{+-}-1-1$ & 3 & & (70) & & & B2 & 2,3 & & & & & \\
\hline & M2 sup. d. & 11 & $\infty 11$ & 11 & & 72 & & & D2 & 2,3 & & 7 & & & \\
\hline & M3 sup. d. & $>3$ & $(\infty)^{3-}-$ & 3 & & (70) & & & $\mathrm{B} 2$ & 2,3 & & & & & \\
\hline \multirow[t]{4}{*}{83.1408} & M2 inf. g. & 12 & $\infty 12$ & 11 & & 72 & & & c & 2,3 & & 7 & XVII & $25-38$ & \\
\hline & M3 inf. g. & $>13$ & $13^{-}$ & 0 & & 85 & & & A & & & & & & \\
\hline & $\mathrm{M} 2$ inf. d. & 13 & $\infty 13$ & 13 & & 72 & & & c & 2,3 & & 7 & & & \\
\hline & M3 inf. d. & $\$ 15$ & $15-$ & 0 & & & & & A & & & & & & \\
\hline \multirow[t]{3}{*}{ Sans $\mathrm{N}^{\circ} 3$} & M2 sup. d. & 11 & $\infty 11$ & 11 & (205) & 75 & & & DI & 2.5 & & 6 & XVIII & $25-38$ & \\
\hline & M3 sup. d. & $?$ & & 0 & & & & & A & & & & & & \\
\hline & M2 sup. g. & 12 & $\Rightarrow 12$ & 12 & & 75 & & & DI & 2.5 & & 6.5 & & & \\
\hline \multirow{4}{*}{$06 \mathrm{HS}$} & $\mathrm{M} 2$ inf. d. & 9 & $\infty 9(x)$ & 9 & & (74) & & & $\mathrm{D} 2$ & 2,5 & & 7 & $\mathrm{xx}$ & $25-38$ & \\
\hline & M3 inf. d. & $>7$ & $\times 7$ & 5 & & (85) & & & B2 & 2,4 & & 8 & & & \\
\hline & $\mathrm{M} 2 \mathrm{inf} \mathrm{p}$. & 9 & $\infty y(x)$ & 9 & & (72) & & & $\mathrm{D} 2$ & 2,5 & & 7 & & & \\
\hline & M3 inf. g. & $>10$ & $\times 10^{-}$ & 3 & & (78) & & & B2 & 2,4 & & 8 & & & \\
\hline \multirow[t]{2}{*}{ Sans $\mathrm{N}^{\circ} 4$} & M2 inf. d. & $>13$ & $\infty 13+$ & 12 & & 70 & & & $\mathrm{C}$ & 2,7 & & 7 & XVII & $25-38$ & \\
\hline & $\mathrm{M} 2 \mathrm{inf}$. g. & $>13$ & $\infty 13+$ & 12 & & 75 & & & c & 2,7 & & 7 & & & \\
\hline \multirow[t]{4}{*}{$89 \mathrm{HS} 033$} & M2 sup. d. & 12 & $\infty 12(\infty)$ & 12 & & 90 & & & $\mathrm{Dl}$ & 2,4 & & 6,5 & XVIII & $25-38$ & \\
\hline & M2 sup. g. & 8 & $-8(\propto)$ & 8 & & 85 & & & D1 & 2,4 & & 6,5 & & & \\
\hline & M3 sup. d. & $?$ & $?$ & 0 & & (95) & & & A & & & & & & \\
\hline & M3 sup. g. & $?$ & $?$ & 0 & & (95) & & & A & & & & & & \\
\hline \multirow[t]{2}{*}{ Sans $\mathrm{N}^{\circ} 5$} & M3 inf. d. & $\rightarrow 11$ & $11+$ & 10 & & 88 & & & $\mathrm{~B}_{3}$ & 2.2 & & 6,5 & $\mathrm{XXI}$ & $25-38$ & M3 inf. g. concrétionnéc \\
\hline & M3 inf, $y$. & $?$ & $?$ & $?$ & & & & & $?$ & 2.2 & & , & $N A$ & 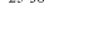 & 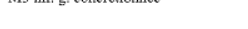 \\
\hline \multirow{5}{*}{ Sans $\mathrm{N}^{\circ} 6$} & Ml inf. g. & 4 & $x 4(x)$ & 4 & & (64) & & & D4 & 2 & & & XVI-XVII & $25-38$ & \\
\hline & M2 inf. g. & 15 & (x) 15 & 14 & & (70) & & & $\mathrm{C}$ & 2 & 8.75 & 9 & & & \\
\hline & M2 inf. d. & 15 & $(x) 15$ & 14 & & (66) & & & c & 2.1 & 7.75 & 8 & & & \\
\hline & M3 inf. g. & 4 & 4. & 0 & & (80) & & & A & & & & & & \\
\hline & M3 inf. d. & 12 & 12. & 0 & & (96) & & & A & & & 8 & & & \\
\hline \multirow[t]{6}{*}{ Sans $\Lambda^{*} 7$} & M1 inf. d. & 6 & $\infty 6(x)$ & 6 & & (59) & & & D3 & 2,5 & & 8 & xv & $13-24$ & \\
\hline & M2 inf. d. & 14 & (x) 14 & 12 & (210) & (71) & & & B4 & 2,2 & & 8 & & & \\
\hline & M1 inf. g. & 5 & $\infty 5(x)$ & 5 & & (57) & & & D3 & 2,5 & & 8 & & & \\
\hline & M2 inf. g. & 14 & (क) 14 & 11 & (215) & (74) & & & B4 & 2,2 & & 8 & & & \\
\hline & M3 inf. d. & $?$ & ?. & 0 & & & & & $\mathrm{~A}$ & & & & & & \\
\hline & M3 inf. g. & $?$ & ?. & 0 & & & & & A & & & & & & \\
\hline \multirow[t]{3}{*}{83.216} & M2 sup. d. & 15 & $\infty 15$ & 13 & (215) & 85 & & & c & 2 & & 7 & XVI-XVII & $25-38$ & 1 lame se divise en 2 cởé vestibulaire \\
\hline & M2 sup. g. & 15 & $\infty 15$ & 13 & (222) & 85 & & & $\mathrm{c}$ & 2 & & 7 & & & \\
\hline & M3 sup. d. & $?$ & $?$ & 0 & & & & & A & & & & & & \\
\hline \multirow[t]{2}{*}{$\operatorname{Sans} \Lambda^{\circ} 18$} & M. inf. g. & $?$ & $\times 7-$ & 0 & & 75 & (145) & 193 & A & & 7.5 & & ? & ? & \\
\hline & M3 sup. d. & $?$ & & 0 & & & & & A & & & & & & \\
\hline Sans $\mathrm{N}^{\circ} 8$ & $\begin{array}{l}\text { M3 supp.g. } \\
\text { M3 sup. d. }\end{array}$ & 20 & $\infty 20-$ & 11 & 375 & 106 & 205 & 192 & $\begin{array}{l}134 \\
134\end{array}$ & 2,9 & $\begin{array}{l}0.23 \\
6\end{array}$ & 5,75 & & $39-4 i$ & \\
\hline
\end{tabular}

\begin{tabular}{|c|c|c|c|c|c|c|c|c|c|c|c|c|c|c|c|}
\hline \multirow{3}{*}{$\frac{N^{2} \text { picec }}{\mathrm{HS} 00140 / 79.249}$} & Photo Position dent & $\mathrm{N}$ & $\mathrm{Nd}$ & $\mathrm{Nf}$ & $\mathrm{Li}$ & 1 & $\mathrm{H}$ & $\mathrm{h}$ & u & c & F lat. & F occl & Laws & Agc approx & Remarques \\
\hline & Fig. 7 M3 inf. g. & $>15$ & $15+$ & 12 & (256) & 84 & & & B4 & 2,5 & & 6,5 & XXIII & $39-47$ & Scule mâchoire entière attcstóc \\
\hline & M3 inf. d. & $>15$ & $15+$ & 12 & (256) & 84 & & & B4 & 2,6 & & & & & \\
\hline & M3 sup. g. & $>14$ & $14+$ & $10-$ & (240) & 85 & & & B4 & ? & & $7 ?$ & & & \\
\hline \multirow[t]{4}{*}{ HSO0146/79,265 } & Fig. 7 MI sup. d. & 8 & $\infty 8(x)$ & 8 & & (69) & & & D3 & 2,7 & & 8,5 & $\mathrm{xV}$ & $13-24$ & \\
\hline & M2 sup. d. & $>13$ & (x) $13+$ & 9 & (200) & (70) & 122 & 174 & B4 & 2,6 & & 9 & & & \\
\hline & Ml sup. g. & 7 & $\infty 7(x)$ & 7 & & (70) & & & $\mathrm{D} 4$ & 2,7 & & 8 & & & \\
\hline & M2 sup. g. & $>13$ & (क) $13+$ & 9 & (187) & (6.3) & 120 & 190 & B34 & 2,6 & & 9 & & & \\
\hline \multirow[t]{2}{*}{ HS00203 } & M. sup d. & 15 & $\circ 15$ & 13 & (230) & 97 & & & c & 2,7 & 7,7 & & XXIV-XXV & $39-47$ & \\
\hline & M1 sup. g. & 15 & ${ }^{\mathrm{D}} 15$ & 12 & (230) & 100 & & & c & 2,7 & 7,7 & & & & \\
\hline \multirow[t]{2}{*}{ HS00147/79.203 } & Fig. 7 M2 sup. d. & 13 & $\infty 13$ & 12 & (185) & 78 & (130) & 167 & c & 2,4 & 7 & 7,5 & $\mathrm{xvI}$ & $25-38$ & La cavité de la M3 g. est présente \\
\hline & M2 sup. g. & 13 & $\approx 13$ & 12 & (185) & 77 & & & c & 2,4 & 7 & 7,5 & & & \\
\hline \multirow[t]{2}{*}{ Sans $\mathrm{N}^{\mathrm{s}} 1$} & MI inf d d & $\geqslant 10$ & $=10+$ & 10 & & 62 & & & $\mathrm{c}$ & 2,3 & 7 & & $\mathrm{XI}$ & $13-24$ & \\
\hline & $\mathrm{M} 1$ inf $g$ & $\geqslant 10$ & $\approx 10+$ & 10 & & 63 & & & $\mathrm{C}$ & 2,3 & 7 & & & & \\
\hline \multirow[t]{4}{*}{$\mathrm{HS} 00283 / 76.375$} & Fig. 7 M2 inf. d. & 11 & $\infty 11(\infty)$ & 11 & & 82 & & & D2 & 2,2 & & 7 & $\mathrm{xXI}$ & $25-38$ & \\
\hline & M3 inf. d. & $>15$ & $15+$ & 9 & & (72) & & & B3 & & & 8,5 & & & \\
\hline & M2 inf. $g$. & 11 & $\infty 11(\alpha)$ & 11 & & 85 & & & D2 & 2,2 & & 7 & & & \\
\hline & M3 inf. $g$. & $>15$ & $15+$ & 9 & & (70) & & & B3 & 2 & & 8,5 & & & \\
\hline \multirow[t]{4}{*}{$\mathrm{HS} 03036$} & $\mathrm{M} 2 \mathrm{inf} \mathrm{d}$. & 13 & $x_{0} 13$ & 12 & & 76 & & & $\mathrm{C}$ & 2,5 & & 6,5 & XVII & $25-38$ & \\
\hline & M2 inf. $\mathrm{g}$. & 13 & $\infty 13$ & 12 & & 78 & & & C & 2,5 & & 6,5 & & & \\
\hline & $\mathrm{M} 3 \mathrm{inl}$ d. & ? & $?$ & 0 & & & & & A & & & & & & \\
\hline & M 3 inl. g. & $?$ & ? & 0 & & & & & A & & & & & & \\
\hline \multirow{4}{*}{ Sans $\mathrm{N}^{\prime \prime} 2$} & M2 sup. d. & 5 & $\infty 5(\infty)$ & 5 & & $(83)$ & & & $\mathrm{D} 3$ & 2,3 & & 6 & $\mathrm{xXI}$ & $25-38$ & \\
\hline & M3 sup. d. & 17 & (ळ) 17 & 6 & 300 & 45 & (180) & 189 & B3 & 2,3 & 7 & 6,5 & & & \\
\hline & M2 sup. g. & 5 & $\infty 5(\infty)$ & 5 & & (83) & & & D3 3 & 2,3 & & 6 & & & \\
\hline & M3 sup. g. & 16 & (ळ) 16 . & 7 & 294 & 100 & (163) & 163 & B3 & 2.3 & 7.25 & 6.5 & & & \\
\hline \multirow[t]{2}{*}{$\mathrm{HS} 00336 / \mathrm{S} 76$} & M2 sup. d. & 10 & $\infty 10(x)$ & 10 & & 92 & & & D1 & 1,8 & & 8 & xVIII & $25-38$ & Contact avec M3 manquantes \\
\hline & M2 sup. g. & 12 & $\infty 12(\infty)$ & 12 & & 93 & & & D1 & 1,8 & & 8 & & & \\
\hline \multirow[t]{4}{*}{83.1107} & M2 inf. d. & 13 & $\infty 13$ & 13 & & 73 & & & D1 & 2,3 & & 7 & $\mathrm{xVIII}$ & $25-38$ & \\
\hline & M3 inf. d. & ? & $x ?$ & 0 & & & & & A & & & & & & \\
\hline & M2 inf. $\mathrm{g}$. & 13 & $\approx 13$ & 13 & & 73 & & & D1 & 2,3 & & 7 & & & \\
\hline & M3 inf $g$. & $?$ & $x ?$ & 0 & & & & & A & & & & & & \\
\hline \multirow[t]{4}{*}{83.166} & M2 sup. d. & $>13$ & $\infty 13+$ & 13 & & 82 & & & $\mathrm{C}$ & 2,4 & & 7,75 & XVII & $25-38$ & 1 lame $M 2$ se divise en 2 côté lingual \\
\hline & M2 sup. g. & $>13$ & 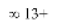 & 13 & & 84 & & & $\mathrm{C}$ & 2,4 & & 7,75 & & & \\
\hline & M3 sup. d. & $?$ & $x ?$ & 0 & & & & & A & & & & & & \\
\hline & M.3 sup. g. & ? & $\mathrm{x}$ ? & 0 & & & & & A & & & & & & \\
\hline \multirow{2}{*}{83.215} & M2 inf. d. & 11 & $x 11$ & 11 & & 68. & & & DI & 1,9 & & 7,5 & $\mathrm{xv}$ & $25-38$ & \\
\hline & M2 inf. g. & 11 & $\approx 11$ & 11 & & 70 & & & D1 & 1,9 & & 7,5 & xVIII & $25-38$ & \\
\hline \multirow{2}{*}{ 962 HS216? } & M2 sup. d. & $>12$ & $\infty 12+$ & 12 & & 76 & & & $\mathrm{c}$ & 2,1 & & 7,5 & xVII & $25-38$ & \\
\hline & M2 sup. g. & $>13$ & $\infty 13+$ & 13 & & 76 & & & c & 2,1 & & 7,5 & & & \\
\hline \multirow[t]{2}{*}{ HSO030 } & $\mathrm{M} 2 / 3 \mathrm{inf} \mathrm{g}$. & $>10$ & $10+$ & 10 & & (70) & & & B4 & 2,3 ? & & 7 & ? & ? & Lecture de $\mathrm{c}$ difficile \\
\hline & $\mathrm{M} 2 / 3$ inf. d. & $>11$ & $11+$ & 11 & & $(70)$ & & & B4 & $2,3 ?$ & & 7 & & & \\
\hline
\end{tabular}




\begin{tabular}{|c|c|c|c|c|c|c|c|c|c|c|}
\hline $\mathrm{N}^{\circ}$ pices & Photo & jugal & Mandibule & Maxillaire & Laws & Age approx & & F lat. & F occl. & Remarques \\
\hline $\mathrm{HS} 06140 / 79.249$ & Fig.7 7 & 3 & M3 (dg) & M3 (g) & XXIII & $39-47$ & 2,6 & & 6,5 & Seule måchoire entière attestré \\
\hline $\mathrm{HS} 00146 / 79.265$ & Fig. 7 & 4 & & $\mathrm{M} 1-\mathrm{M} 2(\mathrm{dg})$ & $\mathrm{xV}$ & $13-24$ & 2,7 & & 9 & \\
\hline IIS06203 & & 2 & & M3 (dg) & $X X I V-X X Y$ & $39-47$ & 2,7 & 7,7 & & \\
\hline HS00147/79.203 & Fig. 7 & 2 & & M2 (dg) & $\mathrm{xVI}$ & $25-38$ & 2,4 & 7 & 7,5 & La cavité de la M3 g. est présente \\
\hline Sans $\mathrm{N}^{\circ} 1$ & & 2 & M1 (dg) & & $\mathrm{XI}$ & $13-24$ & 2,3 & 7 & & \\
\hline Sans $\mathrm{N}^{\circ} 13$ & & $1^{8}$ & M2 (d) & & XVIII & $25-38$ & 2,3 & & 6,5 & \\
\hline HSO01297 & & 1" & & $M(d)$ & ? & & & 7 & & \\
\hline Sans $\mathrm{N}^{\circ} 14$ & & $1^{*}$ & & M & $\mathrm{XVIII-XXX}$ & $>30$ & 2,6 & 7 & & Encore enfouic dans le sédiment \\
\hline 11S012016 & & 1 & & $M(g)$ & $?$ & ? & $?$ & & 7 & Coincée entre maxillaire et sédiment \\
\hline HS00283/76.375 & Fiy. 7 & 3 & $\mathrm{M} 2+\mathrm{M} 3(\mathrm{dg})$ & & $\mathrm{xXI}$ & $25-38$ & 2,2 & & 7,75 & \\
\hline HS03036 & & 3 & $\mathrm{M} 2+\mathrm{M} 3(\mathrm{dg})$ & & xVII & $25-38$ & 2,5 & & 6,5 & \\
\hline $05 \mathrm{HS} 105$ & & $1^{*}$ & & $\mathrm{M}$ & $?$ & & & & & \\
\hline Sans $\wedge 2$ & & 4 & & $\mathrm{M} 2+\mathrm{M} 3(\mathrm{dg})$ & $\mathrm{x} x \mathrm{I}$ & $25-38$ & 2,3 & 7 & 6,25 & \\
\hline HS00336/S 76 & & 2 & & M2 (dg) & XVIII & $25-38$ & 1,8 & & 8 & Contact avec M3 manquante \\
\hline 83.11107 & & 4 & $\mathrm{M} 2+\mathrm{M} 3(\mathrm{dg})$ & & XVIII & $25-38$ & 2,3 & & 7 & \\
\hline 83.166 & & 4 & & $\mathrm{M} 2-\mathrm{M} 3$ (dg) & XVII & $25-38$ & 2,4 & & 7,75 & 1 launc M2 su divise $\mathrm{en} 2$ còté lingual \\
\hline 83.215 & & 2 & M2 (dg) & & XVIII & $25-38$ & 1,9 & & 7,5 & \\
\hline HS00706? & & 1 & & M2 (d) & XVII & $25-38$ & 2,6 & & 7,5 & Scule encore en place dans le maxillaire \\
\hline HS00408 / HSO0082 & & 1 & & M2 (g) & XVIII & $25-38$ & 2,6 & & 6 & Seule encore en place dans le maxillaire \\
\hline $962 \mathrm{HS} 216 ?$ & & 2 & & M2 (dg) & XVII & $25-38$ & 2,1 & & 7,5 & \\
\hline $\mathrm{HS}(\mathrm{H}) 30$ & & 2 & $\mathrm{M} 2: 3$ (dg) & & $x V-x x I I I$ & $24 \mathrm{ou} 41$ & 2.3 & & 7 & Lecture de e difficile \\
\hline $90 \mathrm{HS} 02$ & & $1^{\text {s }}$ & & $\mathrm{M} 2 / 3$ & $?$ & ? & 2.2 & & 7 & En partic masquéc par le sédiment \\
\hline 89115062 & & 4 & & $\mathrm{M} 2+\mathrm{M} 3(\mathrm{dg})$ & $x x$ & $25-38$ & 2,3 & & 7,25 & Cément des M3 important \\
\hline 83.1408 & & 4 & $\mathrm{M} 2+\mathrm{M} 3(\mathrm{dg})$ & & XVII & $25-38$ & 2,3 & & 7 & \\
\hline Sans $\mathrm{N}^{0} 3$ & & 3 & & $\mathrm{M} 2(\mathrm{dg})+\mathrm{M} 3(\mathrm{~d})$ & XVIII & $25-38$ & 2,5 & & 6,25 & \\
\hline $06 \mathrm{HS}$ & & 4 & $\mathrm{M} 2+\mathrm{M} 3(\mathrm{dg})$ & & $\mathrm{xx}$ & $25-38$ & 2.5 & & 7,5 & \\
\hline Sans $\mathrm{N}^{\circ} 4$ & & 2 & M2 (dg) & & xVII & $25-38$ & 2,7 & & 7 & \\
\hline Sans $N^{\circ} 15$ & & 1 & & & XV-XXIII & 24 ou 41 & 2,8 & & 6 & Hémi-mandibule sortant du sédiment \\
\hline 8945033 & & 4 & & $\mathrm{M} 2+\mathrm{M} 3$ (dg) & XVIII & $25-38$ & 2,4 & & 6,5 & \\
\hline Sans $N^{\circ} 5$ & & 2 & M3 (dg) & & $\mathrm{xXI}$ & $25-38$ & 2,2 & & 6,5 & M3 inf. g. concrétionnéc \\
\hline Sans $\mathrm{N}^{\circ} 17$ & & 1 & & M3 & ? & ? & & & & Accessibilitié gênée dans le maxillaire \\
\hline MSL692 & & $1^{*}$ & & M2 (g) & XVI-XVII & $25-38$ & 1,8 & 7 & 7 & Dent casséc. Présence d' 1/2 lame \\
\hline MSL. 727 & & 1 & & M1 (g.?) & $\mathrm{XI}$ & $13-24$ & 1,5 & 9 & 8 & Doute M2.M.primigenius (Lister,1994) \\
\hline MSL739 & & $1^{8}$ & & $\mathrm{M} 2(\mathrm{~g})$ & xIV & $13-24$ & 1,7 & 8,25 & 8 & \\
\hline MSL.157 & & $1^{\circ}$ & & M2 (g) & xVl-xv11 & $25-38$ & 1,4 & 7,5 & 7,5 & Cément important \\
\hline MSL734 & & $1^{*}$ & & M3 (g) & XVII-XVIII & $25 \cdot 38$ & & 6,25 & & \\
\hline MSL. 737 & & 1" & & $\mathrm{M} 2 / 3$ (d) & xyl-xxy & 27 ou 46 & 1,9 & 5,5 & 5,5 & \\
\hline MSL738 & & $1^{*}$ & & M3 (d) & XVII-XVIII & $25-38$ & & & & Dans sa matricc osscusc \\
\hline MSL 735 & & $1^{\prime \prime}$ & & $\mathrm{Ml} / 2(\mathrm{~g})$ & VII-XI & 60015 & & 6,5 & & \\
\hline Sans $\mathrm{N}^{\circ} 18$ & & $1^{*}$ & $M(g)$ & & $?$ & $?$ & & 7,5 & & \\
\hline Sans $\mathrm{N}^{\circ} 12$ & & 2 & $\mathrm{M} 2+\mathrm{M} 3$ (d) & & XVl-XvII & $25-38$ & 1,86 & & 6,75 & \\
\hline MSL736 & & $1^{8}$ & & $M(g)$ & ? & ? & & & & \\
\hline MSL 239 & & $1^{*}$ & & $\mathrm{M}$ & $?$ & $?$ & & & & \\
\hline
\end{tabular}

Tableau 4 - Données obtenues sur les séries dentaires de Mammuthus columbi, Hot Springs.

\begin{tabular}{|c|c|c|c|c|c|c|c|c|c|}
\hline$N^{\circ}$ piece & Photo $\mathrm{Nb}$ jugale: & Mandibule & Maxillaire & Laws & Aye approx & & F lat. & F ovecl. & Remarques \\
\hline MSL119 & $1^{*}$ & & $\mathrm{M} 2 / 3$ & $?$ & $?$ & & & & \\
\hline MSL.120 & $1^{*}$ & & $\mathrm{M} 2 / 3$ & ? & ? & & & & \\
\hline MSL519 & $1^{*}$ & $M(d)$ & & ? & ? & & & & \\
\hline MSL 741 & $1^{*}$ & & $\mathrm{M} 2 / 3(\mathrm{~g})$ & ? & $?$ & 1,7 & 7,5 & 7,5 & \\
\hline MSL 742 & $1^{*}$ & & M3 (d) & $\mathrm{x} \times \mathrm{I}$ & $25-38$ & 1,6 & 8,5 & 8 & \\
\hline MSL 743 & $1^{*}$ & & M3 (g) & $\mathrm{xx}$ & $25-38$ & 1,6 & 8 & 8 & \\
\hline MSL 745 & $1^{*}$ & M3 (d) & & XXVII & $>47$ & 2,6 & & 5,25 & \\
\hline MSL 746 & $1^{*}$ & & $\mathrm{M} 3(\mathrm{~d})$ & XXI & $25-38$ & & 6.25 & 7 & \\
\hline MSL. 749 & $1^{*}$ & & M3 (g) & XXVII & $>47$ & 3 & 4,5 & & Morphologie archaïque \\
\hline MSL 747 & $1^{*}$ & & M2 (d) & XVIII & $25-38$ & 1,6 & 7,5 & 8.5 & \\
\hline MSL 748 & $1^{*}$ & & $M(g)$ & ? & $?$ & & 8 & & \\
\hline MSL 750 & $1^{*}$ & M & & ? & ? & & 9 & & \\
\hline MSL812 & $1^{*}$ & M3 (d) & & $\mathrm{xx}$ & $25-38$ & 1,8 & 6 & 8 & \\
\hline MSL.1083 & $1^{*}$ & & M & $?$ & $?$ & 2,1 & 6 & & \\
\hline MSL1139 & $1^{*}$ & & M2 (g) & $\mathrm{XX1}$ & $25-38$ & 1,8 & 7,5 & 7,5 & \\
\hline MSL531 & $1^{*}$ & & $\mathrm{M}$ & $?$ & ? & 1,4 & & & \\
\hline MSL575 & 1 & & & $?$ & ? & 2 & & & Frayment de lame \\
\hline MSL 576 & 1 & & & $?$ & ? & 1,7 & & & Fragment de lame \\
\hline MSL.1196 & 1 & & & ? & ? & & & & Fragment de lame \\
\hline MSL1197 & 1 & & & $?$ & ? & & & & \\
\hline MSL521 & 1 & & & ? & $?$ & 1,8 & & & \\
\hline IISMS85001 & 1 & & & XIV-xxI & 22 ou 36 & 2 & 9 & 9 & \\
\hline HSMS 85004 & $1^{*}$ & & $\mathrm{M} 2$ (d) & XVI-XVII & $25-38$ & 2,3 & 7 & 7,25 & \\
\hline HSMS76391 & $1^{*}$ & & $\mathrm{M3}(\mathrm{g})$ & XXVII & $>47$ & 2 & 6,75 & 6 & \\
\hline Sans $\mathrm{N}^{\circ} 6$ & 5 & $\mathrm{M} 1(\mathrm{~g})-\mathrm{M} 2+\mathrm{M} 3(\mathrm{dg})$ & & XV1-XVII & $25-38$ & 2,1 & 8,25 & 8,5 & \\
\hline Sans $\mathrm{N}^{\circ} 7$ & 6 & $\mathrm{M} 1+\mathrm{M} 2+\mathrm{M} 3$ (dg) & & $\mathrm{xV}$ & $13-24$ & 2,3 & & 8 & \\
\hline 83.216 & 4 & & $\mathrm{M} 2+\mathrm{M} 3(\mathrm{dg})$ & XVI-XVII & $25-38$ & 2 & & 7 & 1 lame se divise en 2 côté vestibulaire \\
\hline Sans $\mathrm{N}^{\circ} 8$ & 2 & & $\mathrm{M} 3(\mathrm{dg})$ & XXIII & $39-47$ & 2,9 & 6 & 6 & \\
\hline 78.157 & 4 & & $\mathrm{M} 2+\mathrm{M} 3(\mathrm{dg})$ & $x x$ & $25-38$ & 2,4 & 7 & 6,5 & \\
\hline MSL1172 & 4 & $\mathrm{M} 2+\mathrm{M} 3(\mathrm{dg})$ & & XVI-XVII & $25-38$ & 3,2 & 4,25 & 4,75 & Cément important \\
\hline Sans $N^{\circ} 9$ & 4 & $\mathrm{M} 2+\mathrm{M} 3(\mathrm{~g})$ & & XVI-XVII & $25-38$ & 2,4 & & 7 & \\
\hline Sans $\mathrm{N}^{\circ} 16$ & 1 & M3 (d) & & $\mathrm{xxvl}$ & $>47$ & 2,3 & & 6,5 & Seule dans I'hémi-mandibule. Cément + \\
\hline Sans $\mathrm{N}^{\circ} 10$ & 2 & M3 (dg) & & XXIII & $39-47$ & 2,9 & & 5,5 & \\
\hline $87.523 / \# 108$ & 4 & $\mathrm{M} 2+\mathrm{M} 3(\mathrm{dg})$ & & $\mathrm{XXI}$ & $25-38$ & 3,3 & & 6,75 & Cément des M3 important \\
\hline Sans $\mathrm{N}^{\circ} 11$ & 4 & $\mathrm{M} 2+\mathrm{M} 3(\mathrm{dg})$ & & XVIIII & $25-38$ & 2,5 & & 6 & Cément des M2 imporkant \\
\hline MSL. $744 / 85.002$ & 2 & & $\mathrm{M} 2+\mathrm{M} 3(\mathrm{~g})$ & XVI-XVII & $25-38$ & 2,8 & & 7,5 & Cément de la M2 important \\
\hline HS00035 / MSL937 & 3 & $\mathrm{M} 1(\mathrm{~d})+\mathrm{M} 2(\mathrm{dg})$ & & $\mathrm{XVI}$ & $25-38$ & 2,4 & & 8 & Cément des M2 important \\
\hline Sans $\mathrm{N}^{\circ} 19$ & $1^{*}$ & & M3 (g) & $\mathrm{xx}$ & $25-38$ & 1,88 & 6,5 & 8 & \\
\hline "Manfred" & 3 & & M2 (dg) + M3 (g) & XVI-XVII & $25-38$ & 2,4 & & 7 & \\
\hline
\end{tabular}

Légende: $\mathrm{M} 2 / 3$ : M2 (avant dernière molaire) ou $\mathrm{M} 3$ (dernière molaire). $\mathrm{M} 2+\mathrm{M} 3(\mathrm{dg})$ : $\mathrm{M} 2$ et $\mathrm{M} 3$ droites et gauches.

Nombre de jugales : « $1 »$ : dent isolée, « $1^{*} »$ : dent seule en place dans le maxillaire ou la mandibule ; $\mathrm{F}$ : fréquence laminaire (lames sur $10 \mathrm{~cm}$ ), mesure latérale et occlusale.

Légende détaillée des tableaux 3 et 4

Dent isolée / série dentaire :

Dent isolée : n'est plus en place (rien n'est mentionné lorsqu'une seule dent est en place 
dans l'os).

Série : la dent appartient à une série dentaire composée d'au moins deux dents.

Position de la dent :

D2 à D4 sont les dents de lait, M1 à M3 sont les molaires définitives ( $\mathrm{m} 1 \mathrm{à} \mathrm{m} 6$ chez les Anglo-saxons). inf. / sup. / d. / g. : inférieure / supérieure / droite / gauche.

\section{$\mathrm{Nb}$ jugales :}

Nombre de jugales par série dentaire. « 1 » indiquera alors que la dent était isolée, « 1

* » signifie que la dent était seule en place dans le maxillaire ou la mandibule.

\section{Mandibule/Maxillaire :}

M2/3 : M2 (avant dernière molaire) ou M3 (dernière molaire)

M2 + M3 (dg) : M2 et M3 droites et gauches

\section{Nombre de lames :}

$\mathrm{N}$ : nombre total de lames préservées (excepté la lamelle : Maglio, 1973).

Ndlaminaire. Un chiffre précédé de «+ » signifie qu'une partie des lames est inaccessible (cachées par la mâchoire par exemple), la dent possède alors au moins 《国. 》 que des lames ont disparu par contact (pression) entre 2 molaires antérieur à l'éruption (Haynes, 1991), « 酋 » indique une cassure de la dent, « $\mathrm{x}$ » la lamelle (première ou dernière lame appartenant au talon).

$\mathrm{Nf}$ : nombre de lames fonctionnelles (lames ouvertes).

\section{Biométrie et morphologie de la molaire :}

$\mathrm{Li}$ : longueur de la couronne dentaire, en $\mathrm{mm}$. (Li) : mesurée à l'instant T de la vie de la dent, elle est donc inférieure à la longueur de la dent neuve et non usée.

Lo : longueur occlusale, soit la longueur maximale mesurable lorsque la dent est en place dans la mâchoire. Elle n'est donnée qu'à titre indicatif. (Lo) signifie que l'usure de la dent est avancée.

1 : largeur maximale de la dent, en mm / (l) : mesurée sur la surface occlusale.

$\mathrm{H}$ : hauteur de la plus haute lame non usée, en $\mathrm{mm} / \mathrm{h}$ : indice d'hypsodontie $=\mathrm{H} / \mathrm{l} \mathrm{x}$ 100.

$\mathrm{u}$ : stade d'usure de la molaire, fonction de schémas d'usure : $\mathrm{A}$ : dent neuve, $\mathrm{B}:$ nombre croissant de lames en fonction, C : dent en milieu de vie (fonction optimale), D : disparition progressive des lames.

e : épaisseur d'émail en mm (moyenne de plusieurs points de mesure).

$\mathrm{F}$ : fréquence laminaire (nombre de lames présentes sur $10 \mathrm{~cm}$ ). On distinguera la fréquence occlusale (occl.) mesurée sur la table d'abrasion ; fréquence latérale (lat.) mesurée latéralement (Maglio, 1973).

\section{Attribution des âges :}

Laws : Groupes d'âge définis d'après l'étude de mandibules d'éléphants d'Afrique actuels (Laws, 1966). Le groupe II correspond à un âge de 6 mois, III : 1 an, IV : 2 ans, VI : $4+/-1$ ans, VII : $6+/-1$ ans, IX : $10+/-1$ ans, X : $13+/-1$ ans, XI : $15+/-1$ ans, XII : $18+/-1$ ans, XIII : $20+/-2$ ans, ..., XXI : $36+/-2$ ans, XXII $: 39+/-2$ ans, XXIII $: 43+/-2$ ans, XXIV : $45+/-2$ ans, XXVI : $49+/-2$ ans, XXVII : $53+/-2$ ans, XXVIII : $55+/-4$ ans, XXIX : $57+/-4$ ans, XXX : $60+/-4$ ans.

Classe d'âge : Les 30 groupes d'âge de Laws sont ici regroupés ici en 7 classes d'âge : âge inférieur à 2 ans, 2 ans à 5 ans, 6 à 12 ans, 13 à 24 ans, 25 à 36 ans, 37 à 48 ans et 48 ans et plus. 


\section{RÉSUMÉS}

Hot Springs dans le Dakota du Sud est incontestablement l'un des sites les plus riches en vestiges de mammouths in situ jamais mis au jour. Le site daté du Pléistocène supérieur correspond à un ancien marécage issu d'une source artésienne. La source, constamment à $35{ }^{\circ} \mathrm{C}$, était providentielle en période glaciaire pour les animaux qui pouvaient se désaltérer et se repaitre d'une végétation verdoyante typique d'un marécage, et cela en toutes saisons. Toutefois, la source constituait un piège impitoyable pour les animaux qui s'aventuraient dans le lac se terminant en entonnoir dans sa partie submergée. Ainsi, il y a \pm 26000 ans BP (moyenne des datations ${ }^{14} \mathrm{C}$, Laury 1990), nombre d'animaux se sont laissés prendre au piège, essentiellement les mammouths. Afin de comprendre ce phénomène, nous nous sommes attachés à dresser la courbe de mortalité des proboscidiens. Pour ce faire, nous avons attribué à chaque individu une classe d'âge, définie par le rang de chaque molaire, au sein de la série jugale, couplé à son degré d'usure. La révision du profil de mortalité de Mammuthus columbi dressé en 1994, au vu des nouvelles données collectées et présentées ici, indique que le piège a affecté une majorité de sujets adultes. En effet, vingt-deux individus sur une totalité de trente-six identifiés d'après leurs molaires, étaient approximativement âgés de 25 à 38 ans. Le déficit en individus séniles associé au manque de très jeunes mammouths est pour le moins surprenant. Ce profil inhabituel pourrait s'expliquer par l'instabilité de berges abruptes et glissantes se dérobant sous un poids imposant, ne laissant aucune chance aux animaux approchant les cinq tonnes de s'extraire d'un bourbier qui ne leur offrait aucune prise en cas de chute. Il ne semble pas que ce profil résulte de mortalités massives catastrophiques mais plutôt de petits groupes de mâles ou de familles dont les jeunes mammouths, moins lourds, auraient pu réchapper.

Hot Springs, South Dakota, is unmistakably one of the richest sites in mammoth remains ever discovered in situ. The late Pleistocene site corresponds to an ancient swamp coming from an artesian well. The spring, whose temperature was constantly $35^{\circ} \mathrm{C}$, was providential during the glacial period for animals providing a nearby green vegetation all year round. However, the spring was a pitiless trap for animals venturing there, as it ended in a swallow hole in his submerged part. Thus, almost 26,000 years BP, many animals let themselves get trapped, especially mammoths. In order to understand this phenomenon, this work will analyse in the population structure of Proboscids. In order to define some age groups, the dental attrition method was applied, based on the position of each tooth in the cheek teeth series, as well as its worn level. The revision of Mammuthus Columbi mortality profile drawn up in 1994, thanks to the new datas collected on the site and submitted here, indicates that 22 of the 36 subjects identified according to their cheek teeth, were approximately 25-38 years old. The deficit of seniles associated with the lack of young individuals is surprising. The trap affected mostly adult subjects. This unusual population structure characterises no known model. It could be understood by the instability of some banks, steep and slippery, which caved in under the imposing weight of animals of several tonnes, not giving them a chance to extricate from this bog and not offering a foothold in case of fall. The profile doesn't seem to result of catastrophic massive death. It rather affected small groups of males or families excluding young mammoths whose lightness helped them escape this trap.

\section{INDEX}

Mots-clés : Mammuthus columbi, profil de mortalité, molaires, piège naturel, paléoenvironnement, Pléistocène supérieur, Dakota du Sud, États-Unis

Keywords : Mammuthus columbi, Death profile, Cheek teeth, Natural trap, Paleoenvironment, Upper Pleistocene, South Dakota, United States 


\section{AUTEUR}

\section{SOPHIE LOUGUET-LEFEBVRE}

Communauté d'Agglomération du Douaisis - Direction d'Archéologie Préventive, 227, rue Jean

Perrin - ZI Dorignies - FR-59500 Douai hypsodonta@gmail.com

HALMA-IPEL - UMR 8164 (CNRS, Lille 3, MCC) - http://halma-ipel.recherche.univ-lille3.fr/

spip.php?article259 